\title{
THE SEGUE STELLAR PARAMETER PIPELINE. III. COMPARISON WITH HIGH-RESOLUTION SPECTROSCOPY OF SDSS/SEGUE FIELD STARS*
}

\author{
Carlos Allende Prieto ${ }^{1,11}$, Thirupathi Sivarani ${ }^{2}$, Timothy C. Beers ${ }^{2}$, Young Sun Lee ${ }^{2}$, Lars Koesterke $^{3}$, \\ Matthew Shetrone ${ }^{3}$, Christopher Sneden ${ }^{3}$, David L. Lambert ${ }^{3}$, Ronald Wilhelm ${ }^{4}$, Constance M. Rockosi ${ }^{5}$, \\ David K. Lai ${ }^{5}$, Brian Yanny ${ }^{6}$, Inese I. Ivans ${ }^{7}$, Jennifer A. Johnson ${ }^{8}$, Wako Aoki ${ }^{9}$, Coryn A. L. Bailer-Jones ${ }^{10}$, AND \\ PAOLA Re FIORENTIN ${ }^{10}$ \\ ${ }^{1}$ McDonald Observatory and Department of Astronomy, University of Texas, Austin, TX 78712, USA; callende@astro.as.utexas.edu \\ ${ }^{2}$ Department of Physics \& Astronomy, Center for the Study of Cosmic Evolution, and Joint Institute for Nuclear Astrophysics, \\ Michigan State University, East Lansing, MI 48824, USA; thirupathi@pa.msu.edu, beers@pa.msu.edu, lee@pa.msu.edu \\ ${ }^{3}$ McDonald Observatory and Department of Astronomy, University of Texas, Austin, TX 78712, USA; lars@astro.as.utexas.edu, shetrone@astro.as.utexas.edu, \\ chris@astro.as.utexas.edu, dll@astro.as.utexas.edu \\ ${ }^{4}$ Department of Physics, Texas Tech University, Lubbock, TX 79409, USA; ron.wilhelm@ttu.edu \\ ${ }^{5}$ University of California Observatories/Lick Observatory, 1156 High Street, Santa Cruz, CA 95064, USA; crockosi@ucolick.org, david@ucolick.org \\ ${ }^{6}$ Fermi National Accelerator Laboratory, P.O. Box 500, Batavia, IL 60510, USA; yanny@ fnal.gov \\ ${ }^{7}$ The Observatories of the Carnegie Institution of Washington, Pasadena, CA; and Princeton University Observatory, Princeton, NJ, USA; iii@ociw.edu \\ ${ }^{8}$ Department of Astronomy, Ohio State University, Columbus, OH, USA; jaj@astronomy.ohio-state.edu \\ ${ }^{9}$ National Astronomical Observatory, Mitaka, Tokyo 181-8588, Japan; aoki.wako@nao.ac.jp \\ ${ }^{10}$ Max-Planck-Institute for Astronomy, Königstuhl 17, D-69117, Heidelberg, Germany \\ Received 2007 October 31; accepted 2008 August 17; published 2008 October 13
}

\begin{abstract}
We report high-resolution spectroscopy of 125 field stars previously observed as part of the Sloan Digital Sky Survey and its program for Galactic studies, the Sloan Extension for Galactic Understanding and Exploration (SEGUE). These spectra are used to measure radial velocities and to derive atmospheric parameters, which we compare with those reported by the SEGUE Stellar Parameter Pipeline (SSPP). The SSPP obtains estimates of these quantities based on SDSS ugriz photometry and low-resolution $(R \sim 2000)$ spectroscopy. For F- and G-type stars observed with high signal-to-noise ratios $(\mathrm{S} / \mathrm{Ns})$, we empirically determine the typical random uncertainties in the radial velocities, effective temperatures, surface gravities, and metallicities delivered by the SSPP to be $2.4 \mathrm{~km} \mathrm{~s}^{-1}, 130 \mathrm{~K}(2.2 \%), 0.21 \mathrm{dex}$, and 0.11 dex, respectively, with systematic uncertainties of a similar magnitude in the effective temperatures and metallicities. We estimate random errors for lower $\mathrm{S} / \mathrm{N}$ based on numerical simulations.
\end{abstract}

Key words: methods: data analysis - stars: abundances - stars: fundamental parameters - surveys - techniques: spectroscopic

\section{INTRODUCTION}

Starting from the sixth public data release (DR-6; AdelmanMcCarthy et al. 2008), the Sloan Digital Sky Survey (SDSS) provides estimates of the atmospheric parameters for a subset of the stars observed spectroscopically in the survey (those in the approximate range of temperature $4500 \leqslant T_{\text {eff }} \leqslant 7500 \mathrm{~K}$ ). Following completion of the main survey (SDSS-I), the SDSS instrumentation has been devoted to several programs, including the Sloan Extension for Galactic Understanding and Exploration (SEGUE), a massive survey of the stellar content of the Milky Way. Collectively, the suite of computer programs employed to determine atmospheric parameters from SEGUE data is known as the SEGUE Stellar Parameter Pipeline (SSPP). Because each of the public data releases of the SDSS includes and supercedes previous releases, DR-6 also includes atmospheric parameters for archival stellar observations in SDSS-I. These stellar parameters are derived by a series of methods, some of

\footnotetext{
* Based on observations obtained with the Hobby-Eberly Telescope (a joint project of the University of Texas at Austin, Pennsylvania State University, Stanford University, Ludwig-Maximilians-Universität München, and Georg-August-Universität Göttingen), the W. M. Keck Observatory (operated as a scientific partnership among the California Institute of Technology, the University of California and NASA), and the Subaru Telescope (operated by the National Astronomical Observatory of Japan).

${ }^{11}$ Present address: Mullard Space Science Laboratory, University College London, Holmbury St. Mary, Surrey RH5 6NT, UK.
}

which consider purely spectroscopic information (continuumnormalized spectra), solely photometry (available in the survey's ugriz system for all targets), or a combination of photometry and spectroscopy. Paper I in this series describes the SSPP in detail (Lee et al. 2008a). Paper II compares the predictions of the SSPP radial velocities and atmospheric parameters with likely members of Galactic globular and open clusters (Lee et al. 2008b).

The SDSS uses a CCD camera (Gunn et al. 1998) on a dedicated $2.5 \mathrm{~m}$ telescope (Gunn et al. 2006) at Apache Point Observatory, New Mexico, to obtain images in five broad optical bands (ugriz; Fukugita et al. 1996) over approximately $10,000 \mathrm{deg}^{2}$ of the high Galactic latitude sky. The survey dataprocessing software measures the properties of each detected object in the imaging data in all five bands, and determines and applies both astrometric and photometric calibrations (Lupton et al. 2001; Pier et al. 2003; Ivezić et al. 2004). Photometric calibration is provided by simultaneous observations with a 20 inch telescope at the same site (Hogg et al. 2001; Smith et al. 2002; Stoughton et al. 2002; Tucker et al. 2006). A technical summary is provided by York et al. (2000).

SDSS-I and the recently completed SEGUE survey have already built the largest-ever catalog of stars in the Milky Way. To date, this includes photometry in five bands for over 200 million stars and spectroscopy for over 400,000 stars (AdelmanMcCarthy et al. 2008). The SDSS spectrographs deliver a 
Table 1

Observations

\begin{tabular}{lcccccc}
\hline \hline Telescope & Instrument & Resolving Power & Slit Width $(\operatorname{arcsec})$ & Wavelength Coverage $(\mathrm{nm})$ & S/N (per pixel) & No. of Objects \\
\hline HET & HRS & 15,000 & 2.00 & $450-770$ & $20-50$ & 81 \\
Keck I & HIRES & 40,000 & 0.86 & $414-850$ & 80 & 11 \\
Keck II & ESI & 7000 & 0.75 & $380-1000$ & $30-60$ & 25 \\
Subaru & HDS & 50,000 & 0.72 & $300-580$ & 80 & 9 \\
\hline
\end{tabular}

resolving power $\lambda / \mathrm{FWHM} \sim 2000$ over the wavelength range $380-900 \mathrm{~nm}$. Data reduction is fully automated, and the SSPP employs the final products from the SDSS pipeline as input to produce atmospheric parameters (effective temperature, surface gravity, and metallicity) for stars with spectral types A, F, $\mathrm{G}$, and $\mathrm{K}$. The best results are obtained for F- and G-type stars spanning the effective temperature range $5000<T_{\text {eff }}<$ $7000 \mathrm{~K}$

The quality of the SSPP atmospheric parameters is evaluated using different approaches, as already described in Paper I: comparing the parameters with previously published spectral libraries, well-studied open and globular clusters, and with high-resolution observations of field stars. Existing spectral libraries are useful in order to evaluate and calibrate the SSPP methods that rely on spectroscopy alone. Allende Prieto et al. (2006) employed the low-resolution Indo-US library (Valdes et al. 2004), and high-resolution spectra from the Elodie library (Prugniel \& Soubiran 2001) and the $\mathrm{S}^{4} \mathrm{~N}$ archive (Allende Prieto et al. 2004). Because the ugriz system was introduced with the SDSS, the stars included in existing spectral libraries lack photometry in this system. In addition, these are relatively bright stars, typically with $V<14 \mathrm{mag}$, and their fluxes exceed the bright magnitude limit for the SDSS set by the saturation threshold of the detectors at the sidereal driftscan rate of the survey. Obtaining data for these bright stars would require special-purpose observations with a very different instrument configuration that would call into question their value as calibration observations for the otherwise homogeneous imaging survey.

Star clusters provide stringent tests of the SSPP, as the same metallicity should be derived for stars that explore wide ranges of masses and luminosities. Paper II in this series examines SSPP results for likely members of clusters included in DR6. One cannot choose clusters with any given metallicity, but has to take what is provided by nature and accessible from Apache Point. Furthermore, the effective temperatures and surface gravities for the members of any given cluster are very strongly correlated, depending on age and chemical composition. This leads to a patchy coverage of the parameter space. Field stars, however, can be chosen to provide better coverage and, therefore, naturally complement the clusters. Among the stars spectroscopically observed with the SDSS, those in the range $14<V<16.5$ mag can be observed at high resolution with large-aperture telescopes and modest integration times. Due to the vast size of the SDSS stellar sample, these stars can be selected to more uniformly cover the parameter space of stellar properties, and have the additional benefit that photometry is already available for them in the native SDSS system.

This paper, the third in the SSPP series, is devoted to the analysis of over 100 SDSS stars newly observed at high resolution with the Hobby-Eberly, Keck, and Subaru telescopes. Section 2 describes the sample selection and the observations. The determination of radial velocities and atmospheric parameters, based on these observations, is discussed in Sections 3 and 4, respectively. Section 5 describes the results for several well-known standard stars observed with the Hobby-Eberly Telescope. Section 6 compares the parameters derived from high-resolution spectroscopy with those from the SSPP. Section 7 describes numerical experiments that explore how the parameters degrade at lower signal-to-noise ratios (S/Ns). Our conclusions are summarized in Section 8.

\section{OBSERVATIONS}

The majority of the data presented in this paper were obtained with the Hobby-Eberly Telescope (HET; Ramsey et al. 1998), located in West Texas, making use of its High Resolution Spectrograph (HRS; Tull 1998). Additional spectra were obtained with the Keck Observatory, using both the High Resolution Echelle Spectrometer (HIRES; Vogt et al. 1994) and the Echelle Spectrograph and Imager (ESI; Sheinis et al. 2002), and with the Subaru telescope and the High Dispersion Spectrograph (HDS; Noguchi et al. 2002), both located on Mauna Kea, Hawaii. Table 1 summarizes the basic information concerning the spectroscopic observations; more details are provided below.

\subsection{Sample Selection}

Field stars with previous spectroscopic observations from SDSS-I or SEGUE were selected for follow-up spectroscopy at higher resolution. Based on preliminary SSPP atmospheric parameters, targets were initially chosen to span the range $5200<T_{\text {eff }}<7000 \mathrm{~K}, 1.5<\log g<5.5$, and $-2.5<$ $[\mathrm{Fe} / \mathrm{H}]<0.5^{12}$. Our targets are relatively bright; most satisfy $g<15.5$ mag. In addition, a number of cooler red giants were also included in the sample, expanding the initial range of temperatures.

Figure 1 illustrates the coverage of parameter space occupied by our targets. Some 300 stars were placed in the HET queue between 2005 November and 2006 October, despite the fact that time was only allocated for observations of about 100 of them. This over-booking strategy allows for very efficient use of the HET queue schedule (Shetrone et al. 2007). The time on Keck and Subaru was used mainly to increase the target density at low metallicities and cooler temperatures.

\subsection{HET Spectra}

On the HET, a 316 grooves $\mathrm{mm}^{-1}$ cross-dispersing grating, and a $2^{\prime \prime}$ wide slit, collecting $80 \%$ of the light from the $3^{\prime \prime}$ diameter science fibers, were chosen to provide nearly full spectral coverage between 400 and $800 \mathrm{~nm}$ at a resolving power $R=\lambda / \mathrm{FWHM} \simeq 15,000$. Some 280 spectra of 115 stars were obtained. The observations were scheduled at low priority on

\footnotetext{
12 Here and throughout the paper we equate metallicity with iron abundance, and use the bracket notation $[\mathrm{Fe} / \mathrm{H}] \equiv \log \left(\frac{\mathrm{N}(\mathrm{Fe})}{\mathrm{N}(\mathrm{H})}\right)-\left(\frac{\mathrm{N}(\mathrm{Fe})}{\mathrm{N}(\mathrm{H})}\right)_{\odot}$, where $N$ represents number density.
} 


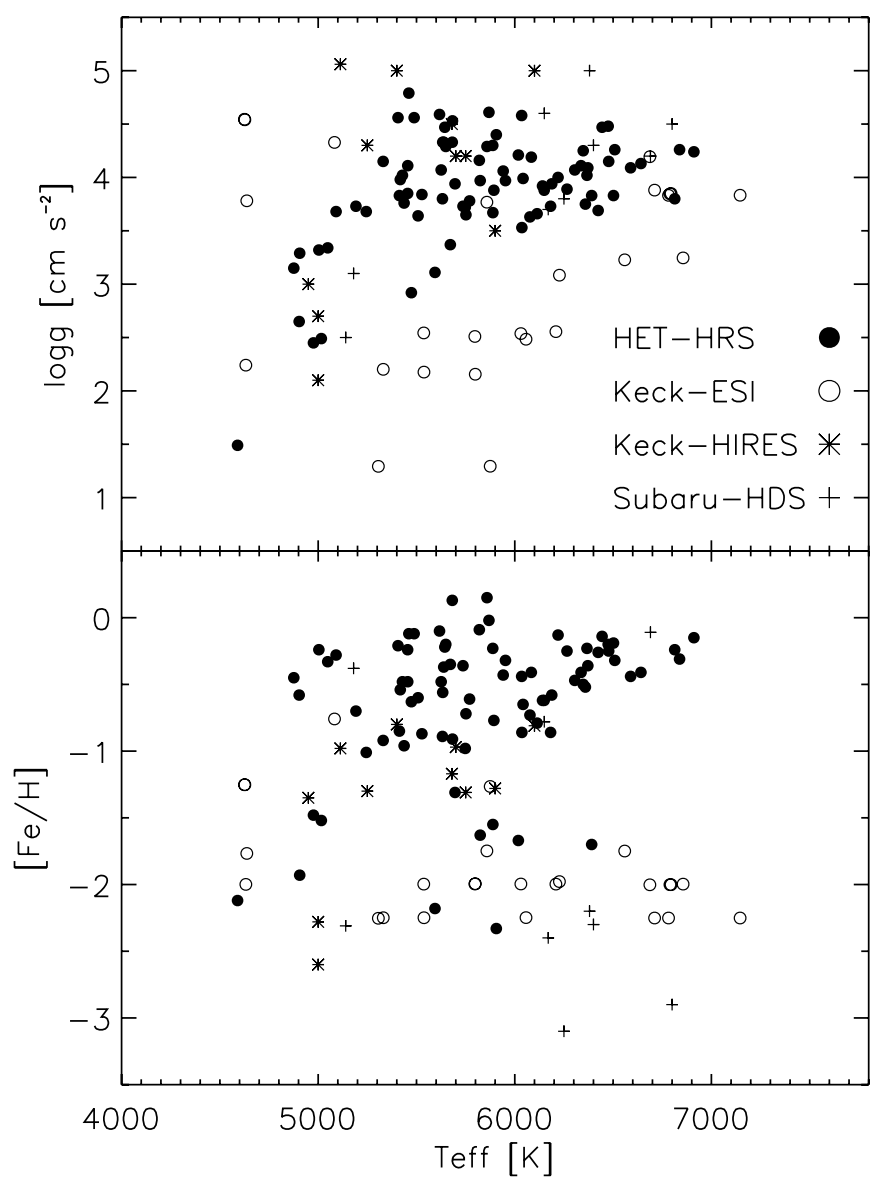

Figure 1. Distribution of the sample of SDSS/SEGUE stars with available high-resolution spectra over the parameter space.

the HET queue, and most were obtained during bright time. Below we discuss only the 81 stars that appeared single-lined, did not exhibit broad lines, and had at least one spectrum with no obvious signs of background light (since no sky fibers were used) and an S/N per pixel at $520 \mathrm{~nm}$ in excess of 20/1.

Data reduction was performed independently at the University of Texas and at Michigan State University (MSU). The reduction at Texas was done automatically, with a pipeline based on IRAF ${ }^{13}$ scripts, while a more interactive procedure, also based on IRAF packages, was employed at MSU. Both reductions included bias removal and flatfield correction, but the former corrected for scattered light with the task apscatter while the latter removed the background for each order from neighboring areas. The results are generally in excellent agreement. Multiple observations were typically obtained for each object. With the exception of nine stars with the lowest $\mathrm{S} / \mathrm{N}$, individual exposures were analyzed independently, and the derived atmospheric parameters were averaged.

\subsection{Keck-HIRES Spectra}

Fourteen objects were observed with the red configuration of the Keck I High Resolution Echelle Spectrometer (HIRESr) and new 3-chip CCD mosaic, with an on-chip binning of $1 \times 2$. The $\mathrm{C} 1$ decker, which has a $7.0 \times 0^{\prime \prime} 861$ slit, was used. This setting

13 IRAF is distributed by the National Optical Astronomy Observatories, which are operated by the Association of Universities for Research in Astronomy, Inc., under cooperative agreement with the National Science Foundation. yields a resolving power of $R \sim 40,000$. The spectra cover a wavelength range of 414-849 $\mathrm{nm}$. Most of the objects had more than two exposures, and exposure times of 300-1500 s. The data were reduced at the Carnegie Observatories, using version 4.0.1. of the Mauna Kea Echelle Extraction data reduction package $\left(\mathrm{MAKEE}^{14}\right)$. The final $\mathrm{S} / \mathrm{N}$ per pixel was approximately $80 / 1$ at $520 \mathrm{~nm}$.

\subsection{Keck-ESI Spectra}

The Keck II ESI spectrograph was used in the echellete mode. Twenty-seven objects were observed with exposure times ranging from 300 to $1200 \mathrm{~s}$. The resolving power is approximately 7000 , using a slit width of 0.74 . The wavelength coverage is $390-1100 \mathrm{~nm}$. Data reduction was performed at Santa Cruz using IRAF scripts (see Lai et al. 2004). The S/N per pixel was in the range $30 / 1-60 / 1$ at $520 \mathrm{~nm}$.

\subsection{Subaru Spectra}

The Subaru HDS was used to observe nine of our program objects with a resolving power of $R \sim 45,000$, covering the wavelength range $300-580 \mathrm{~nm}$. The blue cross disperser was chosen for the observations, with 400 grooves $\mathrm{mm}^{-1}$ and blaze angle of 4.76. Most of the objects had only one exposure. Standard data reduction procedures (bias subtraction, flat-fielding, background subtraction, extraction, and wavelength calibration) were carried out with the IRAF echelle package. Suspected cosmic-ray hits are removed using the technique described by Aoki et al. (2005). The $\mathrm{S} / \mathrm{N}$ per pixel was roughly $80 / 1$ at $520 \mathrm{~nm}$.

\section{RADIAL VELOCITIES}

Following the same strategy as for the data reduction, the radial velocities for HET spectra were measured independently at Texas and MSU by three different methods. There were ten observations of four radial velocity standards, which are discussed in Section 5.

For the Keck-ESI and the MSU reductions of the HET spectra, radial velocities were derived from cross-correlations with the solar spectrum between 480 and $530 \mathrm{~nm}$ (Wallace et al. 1998). After the spectra were analyzed and the atmospheric parameters determined, as explained below (Section 4.2), the cross-correlation was repeated using the best-fitting models as templates. Heliocentric corrections were estimated using the IRAF task rvcor. The radial velocities for the Keck-HIRES data were estimated by cross correlation using the positions of about $100 \mathrm{Fe} \mathrm{I}$ and $10 \mathrm{Fe}$ II lines. Heliocentric corrections were already applied during data reduction by the MAKEE package.

Radial velocities were derived for the Texas-reduced HET spectra by measuring the central wavelengths of several hundred Fe I lines and comparing to laboratory values (Nave et al. 1994). The distribution was then fit by a Gaussian plus a background parabola in order to determine the mean, the sigma, and the error of the mean. The heliocentric correction was estimated with the IRAF task rvcor, then applied in order to obtain the final radial velocity.

The Texas-reduced HET spectra were also cross-correlated with a library of synthetic spectra smoothed to the appropriate resolution in order to measure the Doppler shifts. The library

\footnotetext{
${ }^{14}$ MAKEE was developed by T. A. Barlow for the reduction of Keck I HIRES data taken with the new 3-chip CCD mosaic. It is freely available from the Keck Observatory.
} 
covers a region of $4 \mathrm{~nm}$ around $\mathrm{H} \beta$, and samples uniformly in $T_{\text {eff }}$ the spectral types $\mathrm{F}$ to mid-K ( 4500 to $7500 \mathrm{~K}$ ), with surface gravities $1.0<\log g<5.0$, and metallicities $-2.5<[\mathrm{Fe} / \mathrm{H}]<$ 0.5 . Each synthetic spectrum was cross-correlated with each HET spectrum, and the peak of the cross-correlation was fit with a Gaussian using the IDL routine $x c$ (Allende Prieto 2007). The Doppler shift is estimated as the mean value for the $10 \%$ of the synthetic spectra that best fit the observed spectrum. The heliocentric correction was computed with the routine baryvel (see Stumpff 1980) from the IDL Astronomy User's Library, ${ }^{15}$ and applied. We note that heliocentric corrections derived in this manner differed by those from IRAF's rvcor task by no more than $0.2 \mathrm{~km} \mathrm{~s}^{-1}$.

In summary, three different procedures for radial velocity estimation were applied to the HET spectra: (1) cross-correlation with the solar spectrum in the $480-530 \mathrm{~nm}$ region, (2) direct measurement of the wavelength shifts of atomic iron lines, and (3) cross-correlation with a library of synthetic spectra in the vicinity of $\mathrm{H} \beta$. Cross-correlation with the solar spectrum was the only method applied to the Subaru and Keck spectra. This method and the FeI method agree with one another slightly better than with the third technique (for HET stars): excluding the spectra of SDSS J033530.56-010038 and SDSS $\mathrm{J} 074151.21+275319$, we find an rms scatter of $1.6 \mathrm{~km} \mathrm{~s}^{-1}$. Thus, we adopt the average of these two methods for all HET stars and exclude these two stars in the comparison with the radial velocities from the SSPP. The radial velocities for the HET stars are listed in Table 3; those for the rest of the sample are listed in Table 4.

\section{ANALYSIS}

The majority of our program stars were observed with the HET-HRS using a common setting, but the rest of the spectra from Keck and Subaru fill important gaps in the parameter space. The HET data were analyzed by an automated spectral fitting technique at the University of Texas. The rest of the spectra were analyzed by a second method for automated spectral fitting (Keck-ESI), or by more traditional methods, using line equivalent widths (Subaru-HDS and Keck-HIRES) at MSU. In order to take advantage of both the homogeneity of the HET spectra, and the expanded coverage of the rest of the observations, we separately consider these two data sets, designated below as "HET" and "OTHERS." One star, SDSS J180922.45+223712, was observed with both HET and Subaru.

\subsection{HET Analysis}

The determination of atmospheric parameters for HET spectra at Texas was based on fitting the observations in the range $500-521 \mathrm{~nm}$. This region includes many individual lines, but it is dominated by transitions of neutral iron, calcium, and magnesium. The spectra were first continuum normalized. The search for the optimal solution is based on the Nelder-Mead algorithm (Nelder \& Mead 1965), with model spectra interpolated using a third-order Bezier scheme, but otherwise the same code and strategy as described by Allende Prieto et al. (2006) were employed. The code is also the same as used by the SSPP for the methods described in Section 4.1 of Paper I. The main difference between the ki13 grid used in the SSPP and the one employed here is the spectral resolution, which is now $R=7700$, instead of $R=1000$. The grid models are based on Kurucz

\footnotetext{
15 See http://idlastro.gsfc.nasa.gov/
}

(1993) model atmospheres spanning $4500 \leqslant T_{\text {eff }} \leqslant 7500 \mathrm{~K}$, $1 \leqslant \log g \leqslant 5$, and $-4 \leqslant[\mathrm{Fe} / \mathrm{H}] \leqslant 0.5$, and the spectra are computed adopting very simple (but accurate in the optical) continuum opacities. With only three fitting parameters (effective temperature, surface gravity, and overall metal abundance), a scaled solar composition is implicit in the analysis, considering an enhancement of the $\alpha$ elements for $[\mathrm{Fe} / \mathrm{H}]<0$. Note that the same Nelder-Mead algorithm, but with a different implementation, is used for the analysis of the Keck-ESI data at MSU, as described below.

It should be emphasized that although the HET-HRS spectra have a resolving power of $R=15,000$, the analysis is performed at a lower resolution. By smoothing both the observed and the synthetic spectra to $R=7700$, we effectively eliminate the effects of stellar rotation, and potential variations with time in the PSF of the spectrograph, increasing the original S/N per pixel and speeding up the calculations. The sacrifice in resolution has a negligible impact on the final accuracy of the derived atmospheric parameters, as checked from the analysis of several hundred spectra from the Elodie library at both $R=15,000$ and $R=7700$. Figure 2 illustrates the fits for three program stars and for the metal-poor standard HD 84937, all observed on the HET. The internal consistency of the derived atmospheric parameters for different observations of the same target is excellent, typically $\sigma=32 \mathrm{~K}, 0.05 \mathrm{dex}$, and $0.02 \mathrm{dex}$ for $T_{\text {eff }}, \log g$, and $[\mathrm{Fe} / \mathrm{H}]$, respectively.

The analysis is further simplified by assuming a relationship between the abundance ratio of the $\alpha$ elements to iron and the iron abundance (see Beers et al. 1999; Equation (2) in Allende Prieto et al. 2006), but it is well known that such a relationship does not apply to all stars in the Galaxy. For example, Reddy et al. (2006) find different slopes for the change in $[\alpha / \mathrm{Fe}]$ with $[\mathrm{Fe} / \mathrm{H}]$ for stars in the thin- and thick-disk populations. The halo values are most likely similar to those for the thick disk. Using the average of $[\mathrm{Mg} / \mathrm{Fe}]$, $[\mathrm{Si} / \mathrm{Fe}],[\mathrm{Ca} / \mathrm{Fe}]$, and $[\mathrm{Ti} / \mathrm{Fe}]$, Reddy et al. find that approximately linear trends apply, although they differ somewhat from the relationship adopted in our calculations. Inspection of their fits suggests slopes of $-0.14 \mathrm{dex} / \mathrm{dex}$ and $-0.07 \mathrm{dex} / \mathrm{dex}$, and intercepts at $[\mathrm{Fe} / \mathrm{H}]=0$ of +0.00 and +0.17 , for the thin- and thick-disk populations, respectively. Oxygen may not follow the same behavior (Ramírez et al. 2007), as it appears to exhibit a more pronounced slope for thin-disk stars, but $\mathrm{Mg}$ and $\mathrm{Ca}$ are the relevant elements for the spectral window we are using. The use of a single relationship for all of the $\alpha$ elements is only an approximation.

Our adopted relationship predicts $[\alpha / \mathrm{Fe}]=+0.27,+0.13$, and +0.00 at $[\mathrm{Fe} / \mathrm{H}]=-1.0,-0.5$, and 0.0 , respectively, while the results of Reddy et al. indicate $[\alpha / \mathrm{Fe}]=+0.14,+0.07$, and 0.00 for the thin-disk population, and $[\alpha / \mathrm{Fe}]=+0.24,+0.21$, and +0.17 for the thick-disk population, respectively, at the same metallicities. Halo stars exhibit similar $[\alpha / \mathrm{Fe}]$ ratios as thickdisk stars with $[\mathrm{Fe} / \mathrm{H}]<-0.7$. These differences have only a small impact on our results. The parameters for thin-disk stars with $[\mathrm{Fe} / \mathrm{H}] \sim-1$ (provided they exist), or for thick-disk stars with solar metallicity (provided they exist), would have a maximum systematic error of 0.2 dex in surface gravity and metallicity, and $100 \mathrm{~K}$ in $T_{\text {eff }}$. At the intermediate metallicities where the two populations overlap, errors would amount to about half of the maximum values.

The analysis procedure was tested and calibrated using two spectral libraries from the literature: $\mathrm{S}^{4} \mathrm{~N}$ (Allende Prieto et al. 2004) and the Elodie.3 library (Prugniel \& Soubiran 2001). Our 


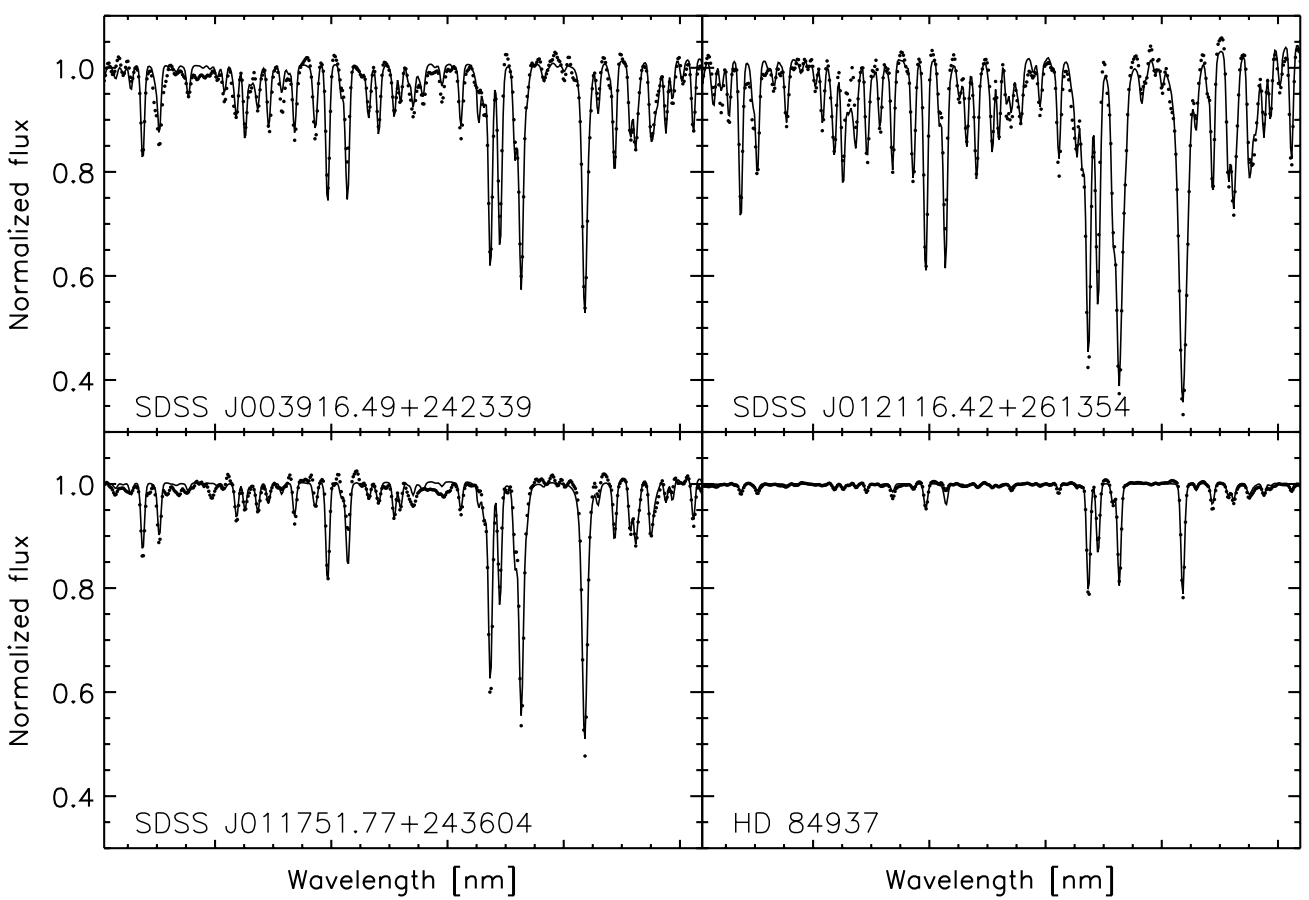

Figure 2. Fittings to individual HET observations for three of the program stars, and the metal-poor standard star HD 84937. The dots correspond to the observations; the solid lines identify the best-fitting model, obtained from cubic Bezier interpolation in the original grid.

Table 2

Average and $1 \sigma$ Scatter of the Differences Between the Parameters Derived (FIT) and Those in the Library Catalogs (LIB), Derived Assuming Gaussian Models

\begin{tabular}{lccc}
\hline \hline Parameter/Library & $\langle$ FIT - LIB $\rangle$ & $\sigma($ FIT - LIB $)$ & $N$ \\
\hline$T_{\text {eff }}-\mathrm{S}^{4} \mathrm{~N}(\mathrm{~K})$ & $-0.10 \%$ & $1.67 \%$ & 55 \\
$\log g-\mathrm{S}^{4} \mathrm{~N}(\mathrm{dex})$ & 0.008 & 0.129 & \\
{$[\mathrm{Fe} / \mathrm{H}]-\mathrm{S}^{4} \mathrm{~N}(\mathrm{dex})$} & -0.001 & 0.049 & \\
$T_{\text {eff }}-$ Elodie $(\mathrm{K})$ & $-2.23 \%$ & $2.66 \%$ & 282 \\
$\log g-$ Elodie (dex) & 0.017 & 0.271 & \\
{$[\mathrm{Fe} / \mathrm{H}]-$ Elodie (dex) } & -0.020 & 0.100 & \\
\hline
\end{tabular}

comparison is limited to stars in these libraries with effective temperatures between $4500<T_{\text {eff }}<7000 \mathrm{~K}$, and, in the case of the Elodie library, with reliable parameters $\left(Q_{\text {Teff }} \geqslant 2\right.$, $Q_{\log g} \geqslant 1$, and $Q_{[\mathrm{Fe} / \mathrm{H}]} \geqslant 3$, where $Q$ represents reliability as defined by the Elodie team). We estimate random and systematic uncertainties by fitting Gaussian models to the differences between the parameters derived for the spectra in these libraries, and their associated catalogs. Our results are systematically different from the $\mathrm{S}^{4} \mathrm{~N}$ catalog parameters by $+5 \%$ in $T_{\text {eff }},+0.20$ dex in $\log g$, and -0.23 dex in $[\mathrm{Fe} / \mathrm{H}]$. After correcting for these zero-point offsets, the differences between our parameters and those in the libraries' catalogs are illustrated in Figure 3; statistics are presented in Table 2, where $\sigma_{\mathrm{rms}}$ is derived from Gaussian fittings.

The larger scatter found for the Elodie library is expected, since the corresponding catalog values do not have a homogeneous source, but are mostly compiled from the literature. In addition, the quality of the original spectra in this library is lower than those in the $\mathrm{S}^{4} \mathrm{~N}$ library. The $1 \sigma$ uncertainties derived from the comparison with the $\mathrm{S}^{4} \mathrm{~N}$ library are adopted as external errors, and added in quadrature to the internal estimates.

The empirically determined corrections from the $\mathrm{S}^{4} \mathrm{~N}$ library for surface gravity and metallicity work as well for the Elodie library. While the first library is dominated by spectra of thindisk stars, the second balances thin-disk, thick-disk, and halo populations, spanning metallicities between -3.0 and +0.5 . With the zero points determined from the comparison with the $\mathrm{S}^{4} \mathrm{~N}$ library, our effective temperatures are roughly $2 \%$ lower than those in the Elodie library. This difference is expected, since the temperatures in the $\mathrm{S}^{4} \mathrm{~N}$ catalog were obtained from the infrared flux method (IRFM) calibrations of Alonso et al. (1996, 1999), while most of the values reported in the Elodie catalog are from spectroscopic analyses. It is well known that the spectroscopic (excitation balance of neutral iron lines, as described in Section 4.2) temperature scale is about $150 \mathrm{~K}$ warmer than the IRFM scale for these spectral types (see Heiter \& Luck 2003; Yong et al. 2004). For consistency with the results of the OTHERS sample, described below, the warmer (Elodie) temperature scale is adopted.

\subsection{OTHERS Analysis}

The atmospheric parameters for the Keck-ESI spectra were derived at MSU, using a grid of synthetic spectra and the IDL optimization routine AMOEBA (see Press et al. 1986), which also employs the Nelder-Mead algorithm.

A total of 13,662 synthetic spectra were generated with a sampling step of $\delta \lambda=5 \times 10^{-4} \mathrm{~nm}$, covering the wavelength range $480-530 \mathrm{~nm}$. The parameter space spans the range 3500 to $9750 \mathrm{~K}$ in $T_{\text {eff }}, 0.0$ to 5.0 in $\log g$, and -2.5 to 0.0 in $[\mathrm{Fe} / \mathrm{H}]$, for $\xi=1,2,3 \mathrm{~km} \mathrm{~s}^{-1}$. The stellar model atmospheres used for the synthetic spectra are the NEWODF models by Castelli \& Kurucz (2003), which include updated opacities for $\mathrm{TiO}$ (Schwenke 1998) and $\mathrm{H}_{2} \mathrm{O}$ (Partridge \& Schwenke 1997). The NEWODF models use solar abundances by Grevesse \& Sauval (1998) and no convective overshooting (Castelli et al. 1997). The synthetic spectra are generated using the turbospectrum synthesis code (Alvarez \& Plez 1998), and employ recent calculations of the broadening of Balmer lines (Barklem et al. 2000) and strong metallic lines (see Barklem \& 

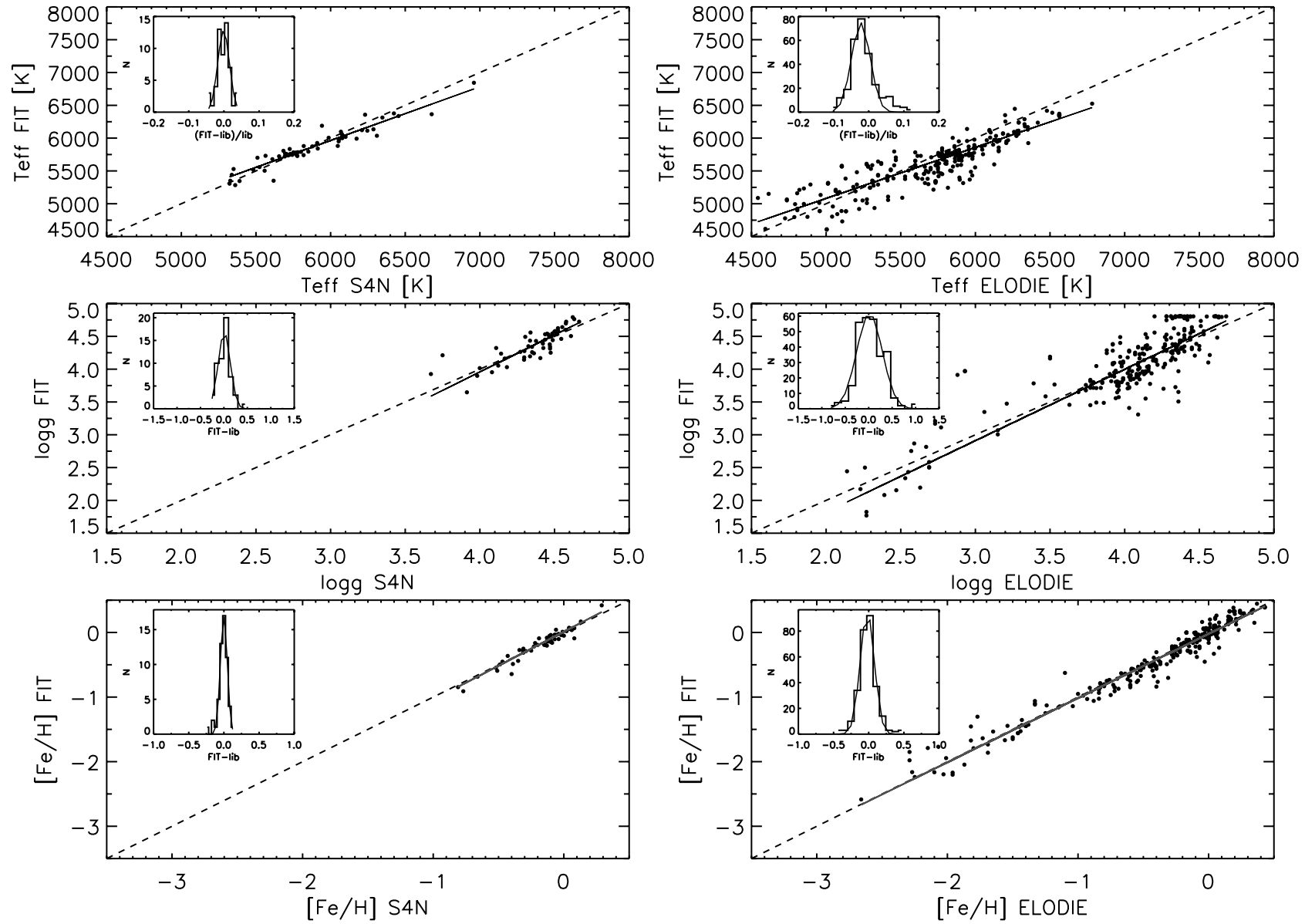

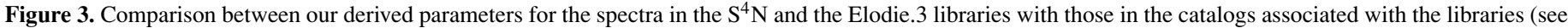

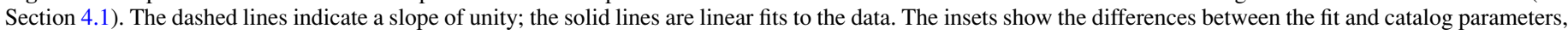
as well as Gaussian models employed to make robust estimates of the median and standard deviation, as shown in Table 2.

Aspelund-Johansson 2005 and references therein) by collisions with hydrogen atoms. The line lists employed come from a variety of sources. Atomic line data are taken mainly from the VALD compilation (Kupka et al. 1999) as of 2002, and in some cases updated from the literature. The atomic line list also includes hyperfine splitting for interesting lines. Line lists for the molecular species $\mathrm{CH}, \mathrm{CN}, \mathrm{TiO}, \mathrm{CaH}$, and $\mathrm{OH}$ were provided by Plez (see Plez 1998; Plez \& Cohen 2005), while the data for the $\mathrm{NH}, \mathrm{C}_{2}$ and $\mathrm{MgH}$ molecules are from Kurucz (see http://kurucz.harvard.edu/LINELISTS/LINESMOL/). The solar abundances compiled by Asplund et al. (2005) were adopted. Finally, the synthetic spectra were reduced to a resolution of $R=7000$ by convolving with a Gaussian. The SSPP parameters were supplied as initial guesses.

The analysis of the Keck-HIRES and Subaru-HDS data was performed at MSU using the equivalent widths of $\mathrm{Fe}$ I and $\mathrm{Fe}$ II lines to constrain $T_{\text {eff }}, \log g,[\mathrm{Fe} / \mathrm{H}]$, and the microturbulence. $T_{\text {eff }}$ is determined from the excitation equilibrium of Fe I lines by forcing a null trend in the excitation potential versus Fe I abundance. $\log g$ is determined from the ionization equilibrium of $\mathrm{Fe} \mathrm{I}$ and Fe II lines. The microturbulence is estimated by forcing a null trend in the equivalent width versus abundance relation. In our analysis we used only lines with equivalent widths $\leqslant 120 \mathrm{~m} \AA$ so as to avoid the nonlinear part of the curve of growth. The atomic data for the Fe I and Fe II lines are from the VALD compilation, and from fits to the solar spectrum. We also checked our estimations by fitting the Balmer line profiles.
We have removed three objects from the Keck-HIRES sample; two of them exhibited very broad lines, apparently due to rapid rotation, while one object was a double-lined spectroscopic binary. For one star, SDSS J205025.83-011103.8, the SSPP did not return measurements.

\section{STANDARD STARS}

The HET sample contains four well-known radial velocity standard stars that have multiple and recent high-resolution analyses in the literature. The stars HD 8648 and HD 84737 have been reported by Nidever et al. (2002) as constant in radial velocity to better than $0.1 \mathrm{~km} \mathrm{~s}^{-1}$ over several years; their heliocentric radial velocities are 0.92 and $4.90 \mathrm{~km} \mathrm{~s}^{-1}$, respectively. Nordström et al. (2004) provide values consistent with these measurements. The radial velocity of HD 71148 has been measured by Nordström et al. to be $-32.6 \pm 0.1 \mathrm{~km} \mathrm{~s}^{-1}$, with consistent measurements reported by Barnes et al. (1986). Nordström also included HD 84937 in their sample, with a radial velocity of $-14.5 \pm 0.2 \mathrm{~km} \mathrm{~s}^{-1}$, in good agreement with previous data from Carney et al. (2001).

The average velocities measured from the HET spectra of HD 8648 (five observations), HD 84737 (one observation), HD 71148 (two observations), and HD 84937 (two observations) are $0.34,4.03,-33.39$, and $-12.31 \mathrm{~km} \mathrm{~s}^{-1}$, respectively. This indicates that a negligible offset exists between the HET values and those adopted from the literature: $-0.01 \pm 0.74 \mathrm{~km} \mathrm{~s}^{-1}$, with an rms scatter of $1.47 \mathrm{~km} \mathrm{~s}^{-1}$. 
Table 3

Comparison of SSPP Velocities and Atmospheric Parameters (HET Sample)

\begin{tabular}{|c|c|c|c|c|c|c|c|c|c|c|c|c|c|c|c|}
\hline \multirow[b]{2}{*}{ Star } & \multirow[b]{2}{*}{ MJD-PLATE-FIBER } & \multicolumn{7}{|c|}{ SSPP } & \multicolumn{7}{|c|}{ HET } \\
\hline & & $V_{R}$ & $T_{\text {eff }}$ & $\sigma$ & $\log g$ & $\sigma$ & {$[\mathrm{Fe} / \mathrm{H}]$} & $\sigma$ & $V_{R}$ & $T_{\text {eff }}$ & $\sigma$ & $\log g$ & $\sigma$ & $\mathrm{Fe} / \mathrm{H}]$ & $\sigma$ \\
\hline SDSS J17 & 51703-0353-605 & -53.60 & 303 & 158 & 4.06 & 0.03 & -0.03 & 0.05 & -50.54 & 5672 & 82 & 3.37 & 0.13 & -0.35 & 05 \\
\hline $801.77+000643.1$ & & 3.80 & 965 & 88 & .97 & .31 & .58 & 0.01 & 3.97 & 6838 & 99 & 4.26 & .13 & & \\
\hline SDSS J010746.51+011402.6 & $51816-0396-605$ & -40.60 & 5682 & 475 & 4.74 & 0.12 & .01 & 0.11 & 43.37 & 5461 & 180 & 4.79 & 0.13 & & 07 \\
\hline SDCC 101440720107202 & & 15.70 & 4715 & 85 & 3.37 & 0.70 & -0.64 & 0.01 & 15.81 & 4876 & 73 & 3.15 & 0.16 & & 06 \\
\hline $5.40+011400.6$ & 517 & 43.50 & & 79 & & 0.12 & -( & 0.10 & 43.68 & 417 & 82 & 3.98 & 0.13 & & .06 \\
\hline $0+011144.9$ & 5187 & -8.90 & 5802 & 60 & 4.56 & 0.11 & -0.12 & 0.09 & -10.17 & 5868 & 87 & 4.61 & 0.13 & & .05 \\
\hline $9+010910.8$ & 62 & -70.20 & 5994 & 61 & 4.36 & 0.12 & & 0.13 & -76.74 & 5527 & 187 & 3.84 & 0.28 & & 16 \\
\hline $26.06+150153.6$ & 51821-0421-439 & -28.10 & 5163 & 28 & 3.62 & 0.73 & -0.32 & 0.07 & -28.51 & 5003 & 162 & 3.32 & 0.45 & & 0.09 \\
\hline SDSS J074705.19+414452.1 & 51885-0434-133 & 73.90 & 5209 & 206 & 3.67 & 0.42 & & 0.11 & & 5048 & 74 & 3.34 & 0.13 & & 0.05 \\
\hline SDSS J082253.87+471742.0 & & -8.00 & 6504 & 111 & 3.36 & 0.63 & & 0.09 & & & 87 & & 0.13 & & 0.05 \\
\hline 10.5 & & & & 130 & & & & & & & 15 & & & & \\
\hline $82+65$ & 5231 & -10.40 & & 74 & & & & & & & 85 & & 0.13 & & \\
\hline & & -3.00 & 6091 & 5 & 33 & & & 0.01 & & 5819 & 2 & & .15 & & 06 \\
\hline & & -58.00 & & 117 & 36 & & & & & & 39 & & 13 & & 05 \\
\hline & & 70 & 174 & 44 & 3.70 & 0.11 & & 0.01 & 19 & 5192 & 77 & & .13 & & .05 \\
\hline & & & & 674 & & & & & & & 325 & & .57 & & 23 \\
\hline & & 0 & & 71 & & 0.73 & & 0.08 & & 5091 & 74 & & .14 & & 05 \\
\hline & & & 24 & 39 & & 0.16 & & & & 44 & 120 & & 0.18 & & 05 \\
\hline & & & & 2 & & 0.12 & & & & 5941 & 86 & & 0.13 & & \\
\hline 3.8 & 15 & -26 & 4 & 38 & 4.24 & 0.11 & & 0 & 2 & 5952 & 86 & 3.97 & .13 & & 05 \\
\hline 3.3 & 78 & -50.60 & 66 & 34 & 4.35 & 0.14 & & 0.07 & & 5638 & 82 & 4.33 & 0.13 & & 05 \\
\hline SD & 52 & 0 & 5729 & 67 & 4.50 & 0.22 & & 0. & & 5644 & 81 & & 0.13 & & 05 \\
\hline & & & & 52 & & & & & & & & & & & \\
\hline & & & & 129 & & & & & & & 92 & & 3 & & \\
\hline & & 40 & & 63 & & 0. & & & 6 & 428 & 283 & & 47 & & 21 \\
\hline & & & & 38 & & & & & & 20 & 90 & & .13 & & 05 \\
\hline & & & 1 & 45 & 3.71 & 0.25 & & 4 & 6 & 38 & 99 & & .14 & & 05 \\
\hline & & & 59 & 51 & 3.84 & 0.09 & -1 & 0.05 & & 18 & 87 & 4.21 & .13 & & 05 \\
\hline & & & & 62 & & 0.09 & & & & 77 & 98 & & 13 & & 05 \\
\hline & & 4.80 & & 61 & & 0.14 & & 0.09 & & 5888 & 101 & 4.30 & .14 & & 05 \\
\hline & & .90 & & 50 & 2.62 & 0.17 & & 0.10 & & 5016 & 72 & 2.49 & 0.13 & & \\
\hline 2026 & 6445 & -138.80 & 5621 & 32 & 3.94 & 0. & -0 & 0.06 & 30 & 5413 & 78 & 3.83 & .13 & & 15 \\
\hline SDSS J004416.51+244246.6 & $53327-2038-154$ & -303.10 & 5594 & 56 & 2.52 & 0.33 & -2.46 & 0.04 & -304.35 & 5594 & 197 & 3.11 & 0.14 & & 0.18 \\
\hline & & & & 38 & & & & & & & 95 & & 0.13 & & \\
\hline & & & & 27 & & & & & & & 8 & & 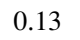 & & \\
\hline & & & & 3 & & & & & & & & & & & \\
\hline & & 30 & & 67 & a & 0 & - & 0 & & 6 & 737 & 3 & 7 & & 8 \\
\hline & & 0 & & 50 & & 0 & & 0.07 & 7 & 4 & 79 & 2 & 3 & & 5 \\
\hline & & 0 & 0 & 41 & 4.22 & 0.07 & $-c$ & 0.30 & 61 & 58 & 87 & 4.29 & 0.13 & 15 & 0.05 \\
\hline & & 0 & & 35 & 4.04 & 0.04 & -0.67 & 0.04 & & 6149 & 129 & 3.88 & 0.18 & 62 & 0.08 \\
\hline & & & & 22 & 3.66 & 0.07 & & & & & 79 & 3.64 & .13 & & .05 \\
\hline & & 26.50 & 6500 & 52 & 4.06 & 0.12 & & 0.12 & & 6305 & 91 & 4.07 & 0.13 & & .05 \\
\hline & -461 & 264.90 & & 93 & 3.80 & 0.26 & -1.86 & 0.11 & & 6391 & 92 & 3.83 & 0.13 & & 5 \\
\hline $.32+222533.4$ & $44-122$ & 77.70 & 5546 & 49 & 4.51 & 0.11 & -0.96 & 0.15 & & 5330 & 79 & 4.15 & 0.14 & & 0.05 \\
\hline SDSS J013924.06+231006.8 & $53327-2044-167$ & -17.80 & 5686 & 29 & 4.19 & 0.12 & -0.34 & 0.10 & -15.74 & 5649 & 99 & 4.29 & 0.15 & & 0.06 \\
\hline & & -145 . & & 92 & & & & 0.26 & 2.72 & 4590 & 66 & 1.49 & 0.13 & & 5 \\
\hline & & & & 3 & & & & & & & 70 & & 8 & & \\
\hline & & & & 10 & & & & & & & 150 & & & & \\
\hline & & 0.60 & 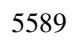 & 52 & 4.11 & 0 . & -0 & 0 & 49 & 5455 & 37 & 3.85 & .06 & & 3 \\
\hline 26.7 & 533 & 33.80 & 6116 & 112 & 4.35 & 0.19 & -0 & 0.12 & 36.93 & 6035 & 27 & 4.58 & 0.15 & $-c$ & .04 \\
\hline $9+351717.7$ & $53446-2$ & 20.90 & 6676 & 47 & 3.91 & 0.13 & -0.50 & 0.05 & 20.14 & 6359 & 338 & 3.75 & 0.56 & -0.52 & .19 \\
\hline $034.52+352545.9$ & $53-130$ & 18.00 & 6279 & 65 & 4.28 & 0.11 & -0.80 & 0.09 & 16.21 & 5893 & 138 & 3.88 & 0.14 & -0.77 & .11 \\
\hline SS J072801.58+354503.3 & $53446-2053-171$ & 9.10 & 6347 & 45 & 3.88 & 0.08 & -0.89 & 0.07 & 9.28 & 6077 & 88 & 3.63 & 0.13 & -0.73 & 0.05 \\
\hline SDSS J072753.81+345437.5 & $53446-2053-226$ & -26.40 & 6790 & 84 & 3.92 & 0.26 & -0.57 & 0.08 & -25.61 & 6589 & 99 & 4.09 & 0.13 & -0.44 & 0.07 \\
\hline $2653.66+370019.9$ & $446-2053-346$ & 30.70 & 6814 & 72 & 4.06 & 0.09 & -0.49 & 0.0 & 33.23 & 6641 & 132 & 4.13 & 0.13 & -0.41 & 0.07 \\
\hline $0.24+370322.8$ & $53446-2053-505$ & -8.30 & 6686 & 66 & 3.86 & 0.28 & -0.47 & 0. & -8.11 & 6508 & 94 & 4.26 & 0.14 & & 0.05 \\
\hline $1512.81+170144.2$ & 524010 & & 6957 & 50 & 3.90 & 0.09 & & 0. & & 6911 & 100 & 4.24 & 0.13 & & 0.05 \\
\hline & & & & 39 & & & & & & & 122 & 3.80 & 0.34 & & 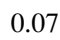 \\
\hline & & & & 34 & & & & & & & 89 & 3.73 & 0.13 & & 5 \\
\hline $112.78+205959.2$ & & 8.50 & 5724 & 61 & 3.54 & 0.15 & & & 33.57 & 770 & 85 & 3.78 & .13 & & .05 \\
\hline $4125.25+212940.8$ & $3378-2078-040$ & 0.20 & 6694 & 48 & 3.91 & 0.13 & & 0.0 & 27.83 & 6424 & 93 & 3.69 & 0.13 & -0.26 & \\
\hline DSS J074017.97+205439.5 & 53378-2078-044 & 11.00 & 6698 & 35 & 3.96 & 0.10 & -0.29 & 0.07 & 7.55 & 6502 & 94 & 3.83 & 0.13 & -0.19 & 0.05 \\
\hline SDSS J073938.60+202314.9 & 53378-2078-049 & 42.00 & 6436 & 92 & 4.01 & 0.16 & -0.65 & 0.07 & 42.37 & 6188 & 89 & 3.94 & 0.13 & -0.58 & 0.05 \\
\hline
\end{tabular}


Table 3

(Continued)

\begin{tabular}{|c|c|c|c|c|c|c|c|c|c|c|c|c|c|c|c|}
\hline \multirow[b]{2}{*}{ Star } & \multirow[b]{2}{*}{ MJD-PLATE-FIBER } & \multicolumn{7}{|c|}{ SSPP } & \multicolumn{7}{|c|}{ HET } \\
\hline & & $V_{R}$ & $T_{\text {eff }}$ & $\sigma$ & $\log g$ & $\sigma$ & {$[\mathrm{Fe} / \mathrm{H}]$} & $\sigma$ & $V_{R}$ & $T_{\text {eff }}$ & $\sigma$ & $\log g$ & $\sigma$ & {$[\mathrm{Fe} / \mathrm{H}]$} & $\sigma$ \\
\hline SDSS J07 & 53378-2078-136 & 14.20 & 6397 & 45 & 3.90 & 0.08 & 0.86 & 0.06 & 12.70 & 6036 & 87 & 3.53 & 0.13 & -0.86 & 0.05 \\
\hline 5.0 & & & 6408 & 12 & 97 & 10 & & & 91 & 13 & 190 & 66 & .25 & & \\
\hline+212940.8 & & 31.60 & 6705 & 52 & 84 & 08 & 27 & & 3.54 & 67 & 92 & 4.02 & .13 & & 05 \\
\hline 244.5 & & 16.90 & 5537 & 38 & 4.58 & 0.09 & & 0.12 & 2.44 & 5617 & 82 & 4.59 & 0.13 & & .05 \\
\hline 08.0 & & 5.40 & 5495 & 46 & 4.17 & 0.09 & & 0.21 & 7.11 & 5682 & 177 & 4.33 & 0.26 & 13 & 12 \\
\hline $30+252145.3$ & & -52.40 & 5913 & 7 & & 0.12 & & 0.10 & & & 85 & 3.72 & 0.14 & & .05 \\
\hline+223712.4 & $4-058$ & 366.10 & 6251 & 75 & 4.00 & 0.27 & & 0.11 & -37 & 5906 & 226 & 4.40 & 0.19 & & 0.15 \\
\hline $36+223720.1$ & $4-083$ & -71.60 & 6065 & 74 & 3.93 & 0.20 & -0.32 & 0.08 & -77.42 & 5736 & 259 & 3.73 & 0.37 & & 0.19 \\
\hline $9924.48+231156.0$ & 534-2184-107 & -200.10 & 5148 & 42 & 2.64 & 0.19 & -1.35 & 0.10 & -195.85 & 4976 & 72 & 2.45 & 0.13 & & 0.05 \\
\hline SDSS J180534.75+244052.7 & 53534-2184-413 & -45.10 & 5489 & 69 & 4.76 & 0.10 & -0.29 & 0.10 & -44.64 & 5488 & 80 & 4.56 & 0.14 & -0.12 & 0.05 \\
\hline SDSS J180418.33+234842.1 & 53534-2184-429 & -166.20 & 6310 & 75 & 4.41 & 0.18 & -1.43 & 0.07 & -170.78 & 5824 & 84 & 3.97 & 0.13 & -1.63 & 0.05 \\
\hline DSS J180623.33+245131.0 & 240184151 & 9.20 & 6274 & 60 & 4.34 & 0.12 & -0.49 & 0. & 3.60 & 6084 & 103 & 4.19 & 0.13 & -0.41 & 0.09 \\
\hline 57.9 & & 54.20 & 5789 & 22 & 4.28 & 0.08 & -0.63 & & 52.61 & 5625 & 277 & 4.07 & 0.43 & -0.48 & 0.22 \\
\hline & & -41.70 & 6103 & 65 & 4. & 0.14 & -1 & 0 . & -47.53 & 5696 & 75 & 3.94 & 0.09 & - & 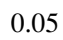 \\
\hline & & 44.20 & 6472 & 86 & 3.5 & 0.10 & -0.40 & 0.08 & 42.07 & 6265 & 131 & 3.89 & 0.18 & -0.25 & 0.06 \\
\hline SDSS J202039.15+140755.2 & $53558-2248-345$ & -30.00 & 5499 & 106 & 4.85 & 0.08 & -0.51 & 0.11 & -30.08 & 5406 & 78 & 4.56 & 0.13 & -0.21 & 0.05 \\
\hline SDSS J220537.22+202904.8 & 53557-2251-305 & 41.70 & 5325 & 38 & 3.54 & 0.18 & -0.99 & 0.07 & 39.62 & 5244 & 77 & 3.68 & 0.14 & -1.01 & 0.05 \\
\hline SDSS J012811.36+385641.0 & 53712-2336-052 & -63.70 & 4737 & 4 & 2.40 & 0.48 & -0.87 & 0.17 & -61.84 & 4903 & 72 & 2.65 & 0.14 & -0.58 & 0.05 \\
\hline
\end{tabular}

HD 8648 has been spectroscopically studied by Mishenina et al. (2004) and Valenti \& Fischer (2005). HD 71148 has been analyzed by Fuhrmann (2004), Lambert \& Reddy (2004), Mishenina et al. (2004), and Valenti \& Fischer (2005). HD 84737 was observed by Chen et al. (2002), Luck \& Heiter (2006), and Valenti \& Fischer (2005). Finally, the spectrum of the halo subdwarf HD 84937 has been analyzed, among others, by Korn et al. (2003), Nissen et al. (2007), and Ryde \& Lambert (2004). The agreement among these studies on the atmospheric parameters for each star is excellent; the rms scatter is less than $80 \mathrm{~K}$ for $T_{\text {eff }}, 0.1 \mathrm{dex}$ for $\log g$, and $0.05 \operatorname{dex}$ for $[\mathrm{Fe} / \mathrm{H}]$. We adopt average values for reference.

A comparison between our adopted literature parameters and those derived from our own analysis of high-resolution HET spectra is provided in Table 5. The effective temperatures of these stars span a limited range, as do their surface gravities, but these objects provide one way to assess the adopted zero points for our atmospheric parameters. On average, our temperatures are $18 \mathrm{~K}$ warmer, our gravities -0.05 dex lower, and our metallicities -0.02 dex lower than the average literature values. These tiny differences indicate no detectable offsets in our derived atmospheric parameters. The rms scatter between our parameters and the literature values are $96 \mathrm{~K}(2 \%), 0.15$ dex, and $0.04 \mathrm{dex}$ in $T_{\text {eff }}, \log g$, and $[\mathrm{Fe} / \mathrm{H}]$, respectively. These estimates are also in excellent agreement with the results based on comparison with the $\mathrm{S}^{4} \mathrm{~N}$ library shown in Table 2. Most of our standard stars have near solar metallicity; the same applies to the stars in the $\mathrm{S}^{4} \mathrm{~N}$ library. However, the agreement with the literature values for $\mathrm{HD} 84937$, at $[\mathrm{Fe} / \mathrm{H}] \simeq-2.1$, for $T_{\text {eff }}$ and $[\mathrm{Fe} / \mathrm{H}]$, does not seem to degrade significantly. The surface gravity, by contrast, does exhibit a larger difference, of about $0.2 \mathrm{dex}$, which suggests a lower precision for this parameter at low metallicity, at least for the HET spectra.

\section{COMPARISON WITH SSPP ESTIMATES}

\subsection{Radial Velocities}

Our two preferred radial velocity determinations for the HET spectra agree with one another with an rms scatter of $1.6 \mathrm{~km} \mathrm{~s}^{-1}$ (an average difference of $0.9 \mathrm{~km} \mathrm{~s}^{-1}$ ). This value is consistent with the scatter inferred for the four radial velocity standards, as described in Section 5. The radial velocities measured in the SDSS spectra that we compare our values with in this section are not derived directly by the SSPP, but, in most cases, they come from matching templates from the Elodie library as part of the spectro-1d pipeline. Nonetheless, the SSPP makes some choices regarding the adopted radial velocity, as explained in Paper I and II, and therefore we refer to the finally adopted radial velocity for the SDSS spectra as the SSPP values below.

The mean S/N per pixel of the SDSS/SEGUE spectra in this set is typically higher than 50/1. The SSPP radial velocities exhibit an rms scatter of $5.1 \mathrm{~km} \mathrm{~s}^{-1}$ relative to the average of our two preferred methods. Nevertheless, this value is not representative for most stars, but it is inflated by three outliers (SDSS J233852.54+140945.7， SDSS J013627.14+231453.6, and SDSS J012106.89+263648.0). A more reliable indication of the typical scatter is derived by least-squares fitting of a Gaussian curve to the differences, which, as Figure 4 illustrates, yields $\sigma=2.9 \mathrm{~km} \mathrm{~s}^{-1}$. This indicates a typical uncertainty of about $2.4 \mathrm{~km} \mathrm{~s}^{-1}$ for the SSPP radial velocities. This level of accuracy is better than in earlier public data releases because of improvements to the DR6 version of the spectro-1d pipeline, primarily to the wavelength solutions, and is consistent with the estimated plate-to-plate scatter in the radial velocity zero point (Adelman-McCarthy et al. 2008).

The SDSS radial velocities involved in our comparison have been systematically corrected by $+7.3 \mathrm{~km} \mathrm{~s}^{-1}$ based on preliminary results from this program, as described by AdelmanMcCarthy et al. (2008), and therefore we limit our discussion to the variance. The unusually large errors for a few stars are likely related to some issue with the SSPP or the SDSS spectra rather than on the HET side. There are a few more examples among the stars observed with KeckI-ESI. The (internal) error bars delivered by the SSPP for the stars in the HET sample range between 0.7 and $2.0 \mathrm{~km} \mathrm{~s}^{-1}$, with a mean value of about $1.3 \mathrm{~km} \mathrm{~s}^{-1}$, or about half our empirical external estimate.

\subsection{Atmospheric Parameters}

The SSPP parameters derived for SDSS spectra discussed in this section are the average values provided as part of SDSS 
Table 4

Comparison of SSPP Velocities and Atmospheric Parameters (OTHERS Sample)

\begin{tabular}{|c|c|c|c|c|c|c|c|c|c|c|c|c|c|c|c|}
\hline \multirow[b]{2}{*}{$\underline{\text { Star }}$} & \multirow[b]{2}{*}{ MJD-PLATE-FIBER } & \multicolumn{7}{|c|}{ SSPP } & \multicolumn{7}{|c|}{ OTHERS } \\
\hline & & $V_{R}$ & $T_{\text {eff }}$ & $\sigma$ & $\log g$ & $\sigma$ & {$[\mathrm{Fe} / \mathrm{H}]$} & $\sigma$ & $V_{R}$ & $T_{\text {eff }}$ & $\sigma$ & $\log g$ & $\sigma$ & {$[\mathrm{Fe} / \mathrm{H}]$} & $\sigma$ \\
\hline \multicolumn{16}{|c|}{ Keck-HIRES } \\
\hline SDSS J131137.14+000803.4 & $51986-0294-623$ & -22.40 & 5060 & 75 & 2.95 & 0.12 & -0.58 & 0.02 & -18.40 & 4950 & 190 & 3.00 & 0.15 & -1.35 & 0.04 \\
\hline SDS & 69 & -48.60 & 5565 & 44 & 2.85 & 0.27 & -1.90 & 0.06 & 60 & 5000 & 70 & 2.70 & 06 & & .01 \\
\hline SDSS J135432.19+000511.3 & 1943-0300-038 & 34.80 & 5898 & 43 & 39 & 0.15 & 0.75 & 10 & 34.50 & 5750 & 100 & 4.20 & 0.08 & & 0.02 \\
\hline & 1942-0301-235 & -27.80 & 5712 & 38 & 4.36 & 0.15 & -0.74 & 0.10 & -21.40 & 5680 & 95 & 4.50 & 0.08 & -1.17 & .02 \\
\hline SDSS J145319.68+010742.5 & 10 & 152.40 & 5422 & 53 & 2.58 & 0.33 & 20 & 0.07 & 148.40 & 5000 & 80 & 2.10 & 0.06 & -2.60 & 0.02 \\
\hline SDSS J004C & $2342-$ & 50.10 & 5254 & 46 & 4.25 & 0.06 & -0.65 & 0.07 & & 5250 & 160 & 4.30 & 0.13 & & 0.03 \\
\hline SDSS $\mathrm{J}$ & 93 & -5.10 & 6271 & 64 & 4.19 & 0.13 & & 0.13 & & 5900 & 115 & 3.50 & 0.13 & & 0.02 \\
\hline & & -48.70 & 5713 & 12 & 4.07 & 0.36 & & 0.15 & -47.20 & 6100 & 145 & 5.00 & 0.12 & & 0.03 \\
\hline & $326-430$ & -0.50 & 5054 & 95 & 4.78 & 0.12 & & 0.13 & & 5113 & 195 & 5.06 & 0.16 & & 0.04 \\
\hline & $786-1328-023$ & -27.00 & 5635 & 14 & 4.56 & 0.13 & & & -26.70 & 5700 & 155 & 4.20 & 0.16 & & 0.03 \\
\hline $\mathrm{SD}$ & 93 & & 5075 & 75 & 4.88 & 0.09 & & & & 5400 & 170 & 5.00 & 0.14 & & 0.03 \\
\hline \multicolumn{16}{|c|}{ Subaru } \\
\hline 5 & 1 & 50.20 & 9 & 93 & 3.70 & 0.36 & 2.18 & 29 & 63 & 00 & 200 & 4.30 & 0.16 & 0 & .02 \\
\hline & & 60 & 6779 & 81 & 3.85 & 0.16 & & 4 & & & 150 & 3.80 & 0.08 & & .02 \\
\hline 0749.9 & 52466 & -419.20 & 6384 & 55 & 3.82 & 0.19 & & 0.09 & 39 & 6170 & 150 & 3.70 & 0.14 & & 0.03 \\
\hline 04.5 & 416 & -66.60 & 5095 & 54 & 2.860 & 0.22 & -0 & 0.08 & 70 & 5180 & 150 & 3.10 & 0.12 & & 0.03 \\
\hline & & 10.30 & 6462 & 183 & 65 & 0.35 & -0.74 & & & 6690 & 130 & 4.20 & 0.10 & & 0.03 \\
\hline 1.9 & 58 & -360 & 6252 & 74 & 04 & 0.33 & -2.21 & 0.11 & 2 & 6380 & 120 & 5.00 & 0.10 & & 0.02 \\
\hline 619.5 & 20 & -337.10 & 5076 & 39 & 2.01 & 0.24 & -2.31 & 0.04 & 18 & 5140 & 95 & 2.50 & 0.08 & 1 & 0.02 \\
\hline & & & & 52 & & & & & -8 & & 65 & & 5 & & 0.01 \\
\hline & & & & 5 & 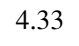 & & & & & 0 & 200 & 0 & 3 & & 0.04 \\
\hline \multicolumn{16}{|c|}{ Keck-ESI } \\
\hline .1 & & .70 & 6747 & 82 & 3.13 & 0.29 & 2.03 & 0.16 & 19.90 & 6856 & 144 & 3.25 & 0.14 & -2.00 & 0.08 \\
\hline & & 2.10 & & & & & & & & 4625 & 148 & 4.55 & 0.29 & & 0.08 \\
\hline 1113.1 & 164 & -22.20 & 4666 & 26 & 4.43 & 0.24 & -1.19 & 0.18 & -23 & 4627 & 172 & 4.54 & 0.34 & & 0.09 \\
\hline $.69+000921.2$ & $84-332$ & -48.30 & 5880 & 26 & 3.73 & 0.41 & -1.87 & 0.08 & -39.81 & 5858 & 104 & 3.77 & 0.13 & -1 & 0.06 \\
\hline $116.19+005913.6$ & $6-557$ & -39.10 & 5982 & 79 & 2.08 & 0.37 & -1.29 & & -45.09 & 5875 & 119 & 1.29 & 0.05 & & 0.05 \\
\hline SDSS J222725.18+003204.6 & 80 & -31.10 & 5953 & 87 & 2.72 & 0.45 & -2.30 & & -34.82 & 6031 & 111 & 2.54 & 0.09 & & 0.07 \\
\hline & & & & 103 & & & & & & 6227 & 116 & & 2 & & 0.07 \\
\hline & & & & 96 & 3.0 & & & & & 37 & 108 & 3.78 & 0.18 & & 0.08 \\
\hline 3.9 & 3 & 228.90 & 6718 & 45 & 3.86 & 0.30 & -2.67 & 0.10 & 240.26 & 6782 & 167 & 3.83 & 0.19 & & 0.11 \\
\hline & & 0 & 6411 & 96 & 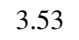 & 0 . & -2 & 0.10 & 4 & 7 & 114 & 4.19 & 0.14 & & 0.07 \\
\hline 1 & 1 & -1 & 5218 & 95 & 4.01 & 0.12 & -0 & 0.09 & -3 & 5083 & 164 & 4.33 & 0.28 & & 0.05 \\
\hline 3.6 & 77 & 10 & 5987 & 46 & 2.81 & 0.45 & -2.25 & 0.13 & 14 & 6209 & 104 & 2.56 & 0.09 & & 0.07 \\
\hline SDSS J002140.87+004820.4 & 53228-1138-391 & -107.70 & 5544 & 68 & 2.36 & 0.39 & -2.41 & 0.08 & -102.12 & 5537 & 92 & 2.54 & 0.09 & -2.00 & 0.07 \\
\hline SDSS J003828.39+003656.6 & 53228-1138-414 & -95.10 & 5815 & 50 & 2.97 & 0.51 & -2.47 & 0.04 & 101.10 & 6057 & 81 & 2.48 & 0.07 & -2.25 & 0.06 \\
\hline 50.4 & & 39.30 & 5824 & 49 & 3.36 & 0.56 & -2.23 & 0.01 & 39.54 & 5797 & 88 & 2.51 & 0.08 & & 0.06 \\
\hline J011135.53-002103.5 & 2592-1143-047 & 137.80 & 6309 & 81 & 3.57 & 0.09 & -2.23 & 0.14 & -171.93 & 6795 & 154 & 3.85 & 0.18 & -2.00 & 0.09 \\
\hline SDSS J020100.13-004259.0 & $3238-1144-402$ & 5.40 & 4721 & 32 & 1.50 & 0.28 & -2.73 & 0.15 & -3.70 & 5331 & 104 & 2.20 & 0.09 & & 0.09 \\
\hline J021748.78+002916.7 & $52992-1485-513$ & -83.90 & 6548 & 47 & 3.51 & 0.45 & -2.32 & 0.20 & -86 & 6711 & 110 & 3.88 & 0.13 & & 0.07 \\
\hline 700 & & & & 33 & 2.3 & & -2 & & & 8 & 85 & 2. & 0.07 & & 0.07 \\
\hline & & -33 & 5649 & 78 & 2.72 & & -2 & & -32 & & 81 & 2.16 & 0.06 & & 0.06 \\
\hline J053234.96-003713.6 & 52944-1495-328 & -92.40 & 6344 & 59 & 3.87 & 0.23 & -2.10 & 0.12 & -92.83 & 6789 & 160 & 3.85 & 0.18 & & 0.10 \\
\hline $22.45+223712.4$ & $52914-14$ & -11.10 & 6330 & 50 & 3.49 & 0.19 & -1.77 & 0.06 & -14.50 & 6559 & 109 & 3.23 & 0.11 & & 0.06 \\
\hline SDSS J181001.41+230554.9 & 52941-1505-094 & -48.00 & 4778 & 198 & 2.67 & 0.95 & -1.64 & 0.92 & -46.50 & 4632 & 157 & 2.24 & 0.15 & -2.00 & 0.14 \\
\hline SDSS J180728.56+223130.5 & 52944-1508-342 & 88.50 & 6695 & 74 & 3.90 & 0.15 & -2.62 & 0.10 & 75.54 & 7146 & 172 & 3.83 & 0.19 & -2.25 & 0.11 \\
\hline SDSS J012617.95+060724.8 & $52945-1521-435$ & -181.50 & 5170 & 45 & 1.83 & 0.13 & -2.42 & 0.05 & -196.13 & 5306 & 102 & 1.29 & 0.05 & -2.25 & 0.09 \\
\hline
\end{tabular}

DR-6 in the public data base (Adelman-McCarthy et al. 2008). In Paper I, we compare the high-resolution parameters against the individual methods integrated into the SSPP in order to estimate their associated systematic and random errors. These will be used in future updates of the SSPP to weight the results from individual methods appropriately.

Figure 5 shows the main result of this paper, the comparison between the estimated stellar atmospheric parameters obtained from the high-resolution spectra with those from the SSPP based on SDSS data. Table 6 summarizes the mean and standard deviation of the differences between the high-resolution results (HI) and those from the SSPP.
There is better agreement between the zero points of the SSPP parameters and the high-resolution results for the OTHERS sample than for the HET results. However, the rms scatter is significantly smaller for the HET sample than for the OTHERS sample. Despite the fact that we have chosen the high (spectroscopic) $T_{\text {eff }}$ scale for calibrating the HET results, we find that the SSPP indicates even higher temperatures, by about 170 $\mathrm{K}$; this value is comparable to the scatter found for this parameter. For SDSS J180922.45+223712, the single star observed both with HET and Subaru, the HET and OTHERS analyses yielded disparate effective temperatures of $5906 \mathrm{~K}$ and $6380 \mathrm{~K}$, surface gravities of $4.40 \mathrm{dex}$ and $5.00 \mathrm{dex}$, and metallicities of -2.33 


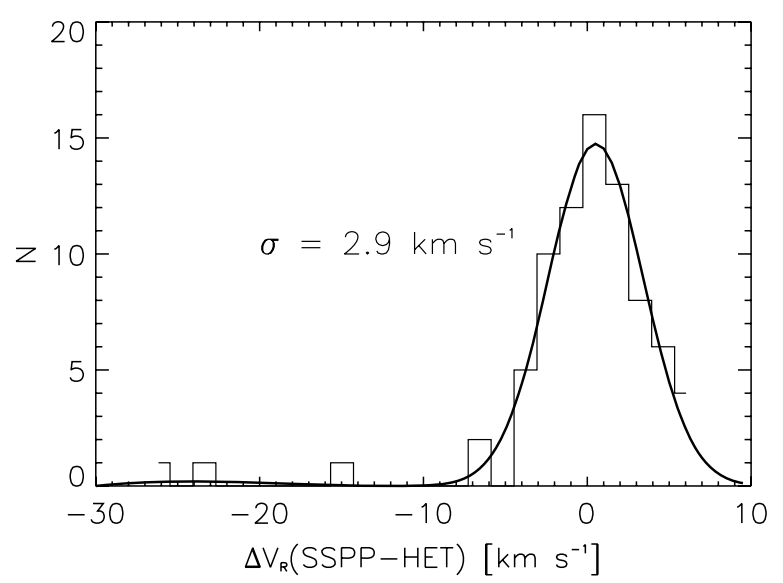

Figure 4. Histogram of the differences between the radial velocities determined by the SSPP from SDSS spectra, and those measured on the HET-HRS spectra. The solid line illustrates a Gaussian model fit by least-squares to the data. The three outliers are SDSS J233852.54+140945.7, SDSS J013627.14+231453.6, and SDSS J012106.89+263648.0.

and -2.20 , respectively. The SSPP $T_{\text {eff }}$ estimate is intermediate to the two values, $6252 \mathrm{~K}$. We note that this is one of the stars with the lowest $\mathrm{S} / \mathrm{N}$ among the HET sample.

The larger scatter for the OTHERS sample is not attributable to the more extended coverage of the parameter space; if we restrict the OTHERS sample to the same range covered by the HET observations, the results do not vary significantly.
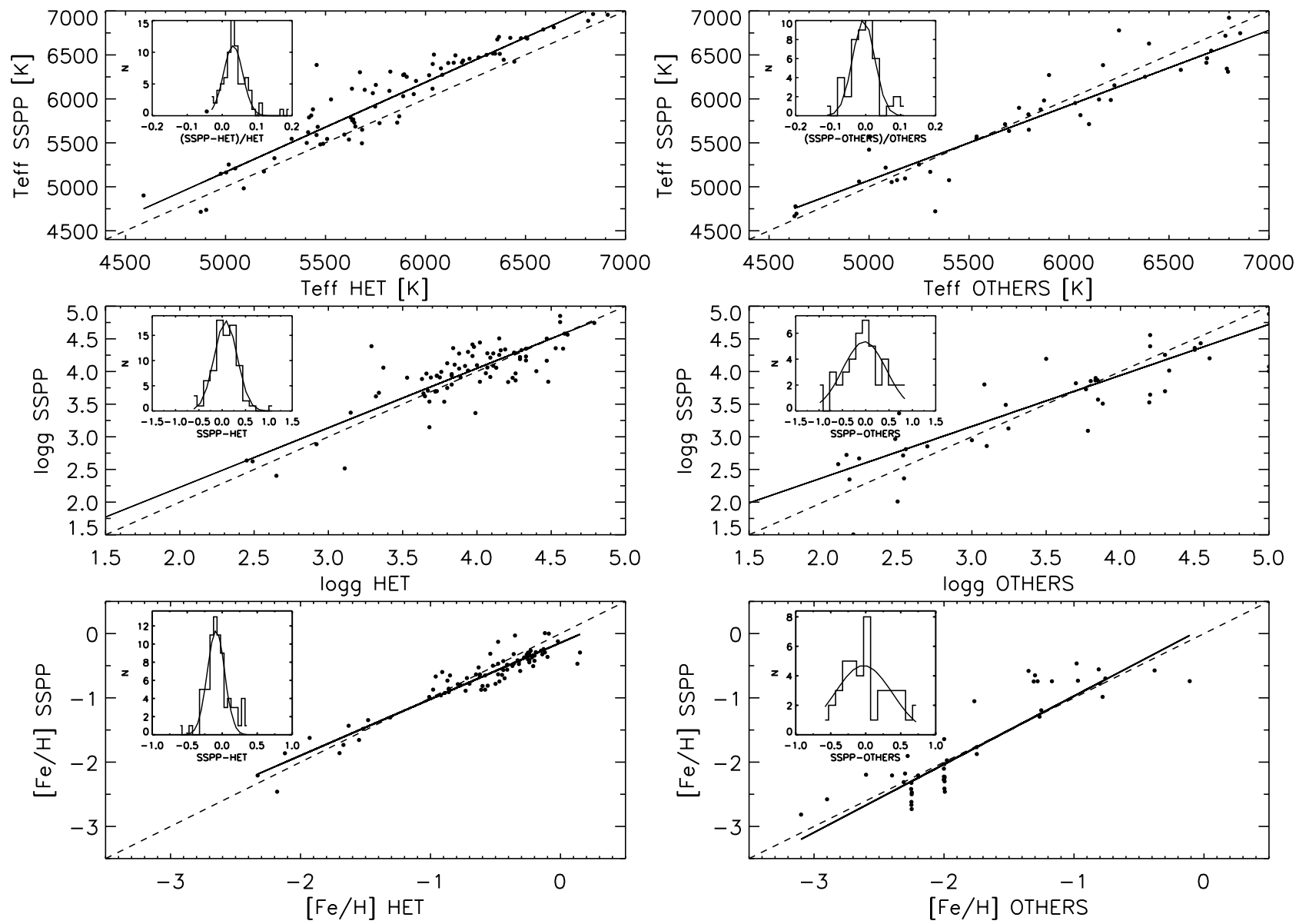

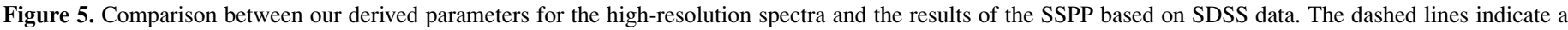

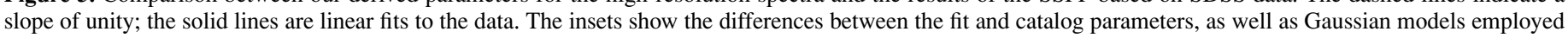
to make robust estimates of the median and standard deviation, as shown in Table 2. 

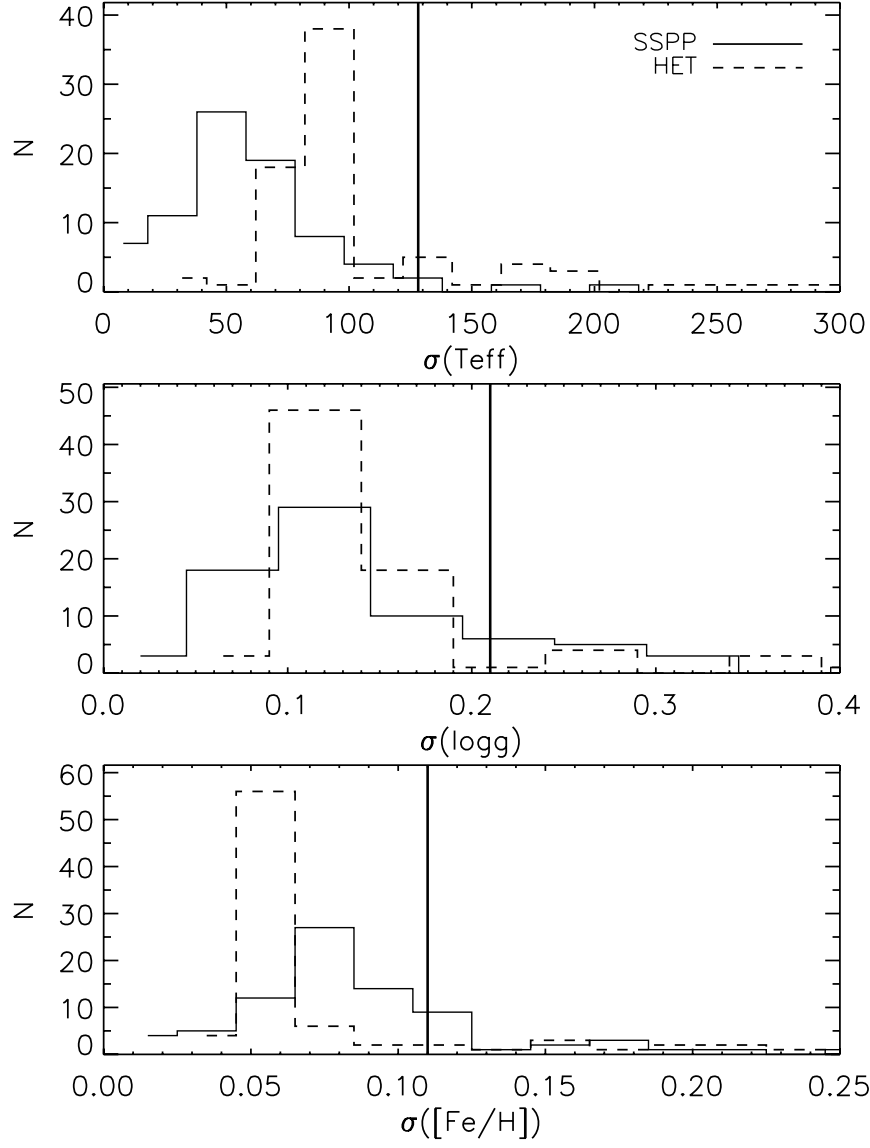

Figure 6. Distribution of estimated (internal) uncertainties in the SSPP parameters (solid lines) and those from high-resolution spectroscopy (dashed) for the HET sample. The vertical lines mark the more realistic external errors for the SSPP parameters, as empirically derived from the comparison with our analysis of HET spectra.

Table 6

Comparison of Derived Stellar Atmospheric Parameters

\begin{tabular}{lcccc}
\hline \hline Analysis & Parameter & $\langle$ SSPP $-\mathrm{HI}\rangle$ & $\sigma(\mathrm{SSPP}-\mathrm{HI})$ & $\mathrm{N}$ \\
\hline HET & Teff $(\mathrm{K})$ & $3.11 \%$ & $2.75 \%$ & 81 \\
& $\log g(\mathrm{dex})$ & 0.08 & 0.25 & \\
OTHERS (Keck, Subaru) & {$[\mathrm{Fe} / \mathrm{H}](\mathrm{dex})$} & -0.09 & 0.12 & \\
& Teff $(\mathrm{K})$ & $-0.58 \%$ & $3.14 \%$ & 44 \\
& $\log g(\mathrm{dex})$ & -0.03 & 0.46 & \\
& {$[\mathrm{Fe} / \mathrm{H}](\mathrm{dex})$} & -0.03 & 0.41 & \\
\hline
\end{tabular}

As previously explained, the uncertainties for the HET spectra are determined by adding in quadrature the uncertainties inferred from the comparison with the $\mathrm{S}^{4} \mathrm{~N}$ library, as shown in Table 2, and the $1 \sigma$ scatter among the values derived from the analysis of individual exposures of each star. The latter contribution is, for most stars, negligible. The SSPP uncertainties correspond to the standard error of the mean $(\sigma / \sqrt{N})$ for the results from the different methods assembled in the pipeline.

Figure 6 shows histograms of the distribution of uncertainty estimates in the HET sample for both the SSPP (solid lines) and the high-resolution HET data (dashed lines). It is unlikely that the parameters obtained from our analysis of high-resolution spectra are more uncertain than those reported by the SSPP. The vertical lines indicate the empirical estimates derived for the SSPP parameters from the comparison with the HET values (see Table 6). The conclusion from this comparison is that the (internal) SSPP error bars significantly underestimate the actual uncertainties, at least for the SDSS/SEGUE spectra with relatively high $\mathrm{S} / \mathrm{Ns}(\mathrm{S} / \mathrm{N}>50 / 1)$. Typically, the quoted SSPP uncertainties in the effective temperature $(\sim 50 \mathrm{~K})$ are about $2-3$ times too small, while those in surface gravity $(\sim 0.12$ dex $)$ and metallicity $(\sim 0.08 \mathrm{dex})$ are about half of their external errors.

\section{UNCERTAINTIES AS A FUNCTION OF S/N}

The comparison in Figure 5 and Table 6 involves a set of SDSS/SEGUE spectra with quite high S/N. Nevertheless, most of the stellar spectra acquired in these projects have a significantly lower $\mathrm{S} / \mathrm{N}$, typically with a wavelength-averaged value of 10/1-20/1. To estimate the effect of a lower $\mathrm{S} / \mathrm{N}$ on the derived atmospheric parameters, we have introduced noise into the original observations.

We followed the same recipe described by Allende Prieto (2007) to create new sets of spectra degraded to an $\mathrm{S} / \mathrm{N}$ at $500 \mathrm{~nm}\left(\mathrm{~S} / \mathrm{N}_{500}\right)$ of $5 / 1,10 / 1,20 / 1$, and $40 / 1$. All sets were analyzed using only one of the methods included in the SSPP: spectral fitting with the ki13 grid, which is described in Paper I (see also Allende Prieto et al. 2006). We found that the derived effective temperatures agree with those determined from HET spectra with an rms scatter of $13 \%, 5 \%, 4 \%$, and $3.2 \%$ at $\mathrm{S} / \mathrm{N}_{500}$ of $5 / 1,10 / 1,20 / 1$, and $40 / 1$, respectively. The derived surface gravities agreed with the high-resolution values with an rms scatter of $0.70,0.55,0.42$, and $0.30 \mathrm{dex}$, while the metallicities agreed with an rms scatter of $0.71 \mathrm{dex}, 0.29 \mathrm{dex}, 0.15 \mathrm{dex}$, and 0.13 dex for a $S / N_{500}$ of $5 / 1,10 / 1,20 / 1$, and $40 / 1$, respectively. Because the kis 13 method and the HET analysis share a number of elements (search algorithm and code, and spectral synthesis data and code), and the spectral windows they exploit overlap, uncertainties could be slightly underestimated at high $\mathrm{S} / \mathrm{N}$, but the figures derived at $S / N=40 / 1$ are in line with those for the original SDSS spectra analyzed with the SSPP (Table 6).

\section{CONCLUSIONS}

We have reported on an analysis of high-resolution spectroscopic observations of a sample of stars previously observed with the SDSS instrumentation as part of SDSS-I or SEGUE. These new data are used to derive radial velocities and atmospheric parameters, and to examine the performance of the SSPP Pipeline described in Paper I in this series. The sample we have examined includes 81 stars observed with the HET-HRS, 25 stars obtained with Keck-ESI, 11 stars observed with Keck-HIRES, and 9 stars from Subaru-HDS.

Through a comparison with external spectroscopic libraries, and by employing multiple methods of analysis for the HET sample, we estimate that our reference radial velocities are accurate to $1.6 \mathrm{~km} \mathrm{~s}^{-1}$. Our values for the stellar atmospheric parameters, effective temperature, surface gravity, and metallicity, are accurate to $1.5 \%(\sim 90 \mathrm{~K}), 0.13$ dex and 0.05 dex, respectively. These figures are derived from the comparison with the parameters for nearby stars in the $\mathrm{S}^{4} \mathrm{~N}$ catalog, but we find they are still valid for the moderately high $\mathrm{S} / \mathrm{N}$ of the HET spectra. Using the HET sample to benchmark the SSPP, subtracting in quadrature the uncertainties in the results for the former, we conclude that the SDSS/SEGUE radial velocities are typically accurate to $2.4 \mathrm{~km} \mathrm{~s}^{-1}$ for high S/N SDSS spectra $(\mathrm{S} / \mathrm{N}>50 / 1)$. A similar comparison of the atmospheric parameters returned by the SSPP with those obtained from HET spectra leads to the conclusion that the SSPP effective temperatures, surface gravities, and metallicities for bright targets show 
random errors of $2.2 \%(\sim 130 \mathrm{~K}), 0.21 \mathrm{dex}$, and $0.11 \mathrm{dex}$, respectively. Systematic offsets of a similar size are detected for the effective temperatures and metallicities. We evaluate the expected random uncertainties as a function of $\mathrm{S} / \mathrm{N}$ by repeating the analysis after introducing noise in the SDSS spectra. More extended tests are under way and will be reported elsewhere.

Our study also finds that the internal uncertainties delivered by the SSPP for both radial velocities and atmospheric parameters need to be systematically increased by a factor of 2-3 in order to be consistent with our derived external errors. The uncertainties in the average SSPP atmospheric parameters are simply derived as the standard error of the mean for a Gaussian distribution from the multiple techniques applied to any particular target. The fact that many methods share the same spectroscopic indicators (e.g., Balmer lines or SDSS color indices to gauge $T_{\text {eff }}$ ), and models (e.g., Kurucz's model atmospheres) may cause unaccounted correlations that result in underestimated uncertainties.

The validation and calibration of the SSPP is an ongoing project. Several additional open and globular clusters have recently had data obtained with SDSS instrumentation, and will be considered in future papers. A sample of up to several hundred very low metallicity stars from SDSS/SEGUE is currently being observed with the HET and Subaru telescopes, and will add to our calibration sample. Additional stars of intermediate metallicity, and with hotter and cooler temperatures than considered in the present work, will also be included in our calibration sample based on observations with a number of large-aperture telescopes. Our goal is to produce an SSPP validation catalog of on the order of 500 stars, which will be used to refine and adjust the individual parameter estimation techniques employed by the SSPP, and thus establish a definitive atmospheric parameter estimation scale for application to the large SDSS/SEGUE stellar samples, as well as to future surveys.

Funding for the SDSS and SDSS-II has been provided by the Alfred P. Sloan Foundation, the Participating Institutions, the National Science Foundation, the U.S. Department of Energy, NASA, the Japanese Monbukagakusho, the Max Planck Society, and the Higher Education Funding Council for England. The SDSS Web site is http://www.sdss.org/.

The SDSS is managed by the Astrophysical Research Consortium for the Participating Institutions. The Participating Institutions are the American Museum of Natural History, Astrophysical Institute Potsdam, University of Basel, University of Cambridge, Case Western Reserve University, University of Chicago, Drexel University, Fermilab, the Institute for Advanced Study, the Japan Participation Group, Johns Hopkins University, the Joint Institute for Nuclear Astrophysics, the Kavli Institute for Particle Astrophysics and Cosmology, the Korean Scientist Group, the Chinese Academy of Sciences (LAMOST), Los Alamos National Laboratory, the Max-PlanckInstitute for Astronomy (MPIA), the Max-Planck-Institute for Astrophysics (MPA), New Mexico State University, Ohio State University, University of Pittsburgh, University of Portsmouth, Princeton University, the United States Naval Observatory, and the University of Washington.

The HET is a joint project of the University of Texas at Austin, the Pennsylvania State University, Stanford University, Ludwig-Maximilians-Universität München, and Georg-AugustUniversität Göttingen. The HET is named in honor of its principal benefactors, William P. Hobby and Robert E. Eberly. Some of the data presented herein were obtained at the W.M. Keck Observatory, which is operated as a scientific partnership among the California Institute of Technology, the University of California, and NASA. The Observatory was made possible by the generous financial support of the W.M. Keck Foundation. The authors recognize and acknowledge the very significant cultural role and reverence that the summit of Mauna Kea has always had within the indigenous Hawaiian community. We are most fortunate to have the opportunity to conduct observations from this mountain.

NASA grants (NAG5-13057, NAG5-13147) to C.A.P. and D.L.L. are thankfully acknowledged. T.C.B., Y.S.L., B.M., and T.S. acknowledge support from the U.S. National Science Foundation under grants AST 04-06784 and AST 07-07776, as well as from grant PHY 02-16783; Physics Frontier Center/Joint Institute for Nuclear Astrophysics (JINA). D.L.L.'s research is supported in part by the Welch Foundation of Houston, Texas.

\section{REFERENCES}

Adelman-McCarthy, J. K., et al. 2008, ApJS, 175, 297

Allende Prieto, C. 2007, AJ, 134, 1843

Allende Prieto, C., Barklem, P. S., Lambert, D. L., \& Cunha, K. 2004, A\&A, 420, 183

Allende Prieto, C., Beers, T. C., Wilhelm, R., Newberg, H. J., Rockosi, C. M., Yanny, B., \& Lee, Y. S. 2006, ApJ, 636, 804

Alonso, A., Arribas, S., \& Martinez-Roger, C. 1996, A\&A, 313, 873

Alonso, A., Arribas, S., \& Martínez-Roger, C. 1999, A\&AS, 140, 261

Alvarez, R., \& Plez, B. 1998, A\&A, 330, 1109

Aoki, W., et al. 2005, ApJ, 632, 611

Asplund, M., Grevesse, N., \& Sauval, A. J. 2005, in ASP Conf. Ser. 336, Cosmic Abundances as Records of Stellar Evolution and Nucleosynthesis, ed. T. G. Barnes, III \& F. N. Bash (San Francisco, CA: ASP), 25

Barklem, P. S., \& Aspelund-Johansson, J. 2005, A\&A, 435, 373

Barklem, P. S., Piskunov, N., \& O’Mara, B. J. 2000, A\&A, 363, 1091

Barnes, T. G., III, Moffett, T. J., \& Slovak, M. H. 1986, PASP, 98, 223

Beers, T. C., Rossi, S., Norris, J. E., Ryan, S. G., \& Shefler, T. 1999, AJ, 117, 981

Carney, B. W., Latham, D. W., Laird, J. B., Grant, C. E., \& Morse, J. A. 2001, AJ, 122, 3419

Castelli, F., Gratton, R. G., \& Kurucz, R. L. 1997, A\&A, 318, 841

Castelli, F., \& Kurucz, R. L. 2003, in IAU Symp. 210, Modelling of Stellar Atmospheres, ed. N. Piskunor, W. W. Weiss, \& D. F. Gray (Dordrecht: Kluwer), 20P

Chen, Y. Q., Nissen, P. E., Zhao, G., \& Asplund, M. 2002, A\&A, 390, 225

Fuhrmann, K. 2004, Astron. Nachr., 325, 3

Fukugita, M., Ichikawa, T., Gunn, J. E., Doi, M., Shimasaku, K., \& Schneider, D. P. 1996, AJ, 111, 1748

Grevesse, N., \& Sauval, A. J. 1998, Space Sci. Rev., 85, 161

Gunn, J. E., et al. 1998, AJ, 116, 3040

Gunn, J. E., et al. 2006, AJ, 131, 2332

Heiter, U., \& Luck, R. E. 2003, AJ, 126, 2015

Hogg, D. W., Finkbeiner, D. P., Schlegel, D. J., \& Gunn, J. E. 2001, AJ, 122, 2129

Ivezić, Ž., et al. 2004, Astron. Nachr., 325, 583

Korn, A. J., Shi, J., \& Gehren, T. 2003, A\&A, 407, 691

Kupka, F., Piskunov, N., Ryabchikova, T. A., Stempels, H. C., \& Weiss, W. W. 1999, A\&AS, 138, 119

Kurucz, R. 1993, Kurucz CD-ROM No. 8 (Cambridge: Smithsonian Astrophysical Observatory)

Lai, D. K., Bolte, M. A., Johnson, J. A., \& Lucatello, S. 2004, AJ, 128, 2402

Lambert, D. L., \& Reddy, B. E. 2004, MNRAS, 349, 757

Lee, Y. S., et al. 2008a, AJ, 136, 2022 (Paper I)

Lee, Y. S., et al. 2008b, AJ, 136, 2050 (Paper II)

Luck, R. E., \& Heiter, U. 2006, AJ, 131, 3069

Lupton, R., Gunn, J. E., Ivezić, Ž., Knapp, G. R., \& Kent, S. 2001, in ASP Conf. Ser. 238, Astronomical Data Analysis Software and Systems X, ed. F. R. Harnden, Jr., F. A. Primini, \& H. E. Payne (San Francisco, CA: ASP), 269

Mishenina, T. V., Soubiran, C., Kovtyukh, V. V., \& Korotin, S. A. 2004, A\&A, 418,551

Nave, G., Johansson, S., Learner, R. C. M., Thorne, A. P., \& Brault, J. W. 1994, ApJS, 94, 221

Nelder, J., \& Mead, R. 1965, Computer Journal, 7, 308

Nidever, D. L., Marcy, G. W., Butler, R. P., Fischer, D. A., \& Vogt, S. S. 2002, ApJS, 141, 503 
Nissen, P. E., Akerman, C., Asplund, M., Fabbian, D., Kerber, F., Kaufl, H. U., \& Pettini, M. 2007, A\&A, 469, 319

Noguchi, K., et al. 2002, PASJ, 54, 855

Nordström, B., et al. 2004, A\&A, 418, 989

Partridge, H., \& Schwenke, D. W. 1997, J. Chem. Phys., 106, 4618

Pier, J. R., Munn, J. A., Hindsley, R. B., Hennessy, G. S., Kent, S. M., Lupton, R. H., \& Ivezić, Ž. 2003, AJ, 125, 1559

Plez, B. 1998, A\&A, 337, 495

Plez, B., \& Cohen, J. G. 2005, A\&A, 434, 1117

Press, W. H., Flannery, B. P., Teukolsky, S. A., \& Vetterling, W. T. 1986, Numerical Recipes (Cambridge: Cambridge Univ. Press)

Prugniel, P., \& Soubiran, C. 2001, A\&A, 369, 1048

Ramírez, I., Allende Prieto, C., \& Lambert, D. L. 2007, A\&A, 465, 271

Ramsey, L. W., et al. 1998, in SPIE Conf. Proc. 3352, Advanced Technology Optical/IR Telescopes VI, ed. L. M. Stepp (Bellingham, WA: SPIE), 34

Reddy, B. E., Lambert, D. L., \& Allende Prieto, C. 2006, MNRAS, 367,1329

Ryde, N., \& Lambert, D. L. 2004, A\&A, 415, 559

Schwenke, D. W. 1998, Chemistry and Physics of Molecules and Grains in Space. Faraday Discussions No., 109, 321
Sheinis, A. I., Bolte, M., Epps, H. W., Kibrick, R. I., Miller, J. S., Radovan, M. V., Bigelow, B. C., \& Sutin, B. M. 2002, PASP, 114, 851

Shetrone, M., et al. 2007, PASP, 119, 556

Smith, J. A., et al. 2002, AJ, 123, 2121

Stoughton, C., et al. 2002, AJ, 123, 485

Stumpff, P. 1980, A\&AS, 41, 1

Tucker, D. L., et al. 2006, Astron. Nachr., 327, 821

Tull, R. G. 1998, in SPIE Conf. Proc. 3355, Optical Astronomical Instrumention, ed. S. D'odorico (Bellingham, WA: SPIE), 387

Valdes, F., Gupta, R., Rose, J. A., Singh, H. P., \& Bell, D. J. 2004, ApJS, 152, 251

Valenti, J. A., \& Fischer, D. A. 2005, ApJS, 159, 141

Vogt, S. S., et al. 1994, in SPIE Conf. Proc. 2198, Instrumentation in Astronomy VIII, ed. D. L. Crawford \& E. R. Craine (Bellingham, WA: SPIE), 362

Wallace, L., Hinkle, K., \& Livingston, W. 1998, An Atlas of the Spectrum of the Solar Photosphere from 13,500 to $28,000 \mathrm{~cm}^{-1}$ (3570 to $7405 \AA$ ) (Tucson, AZ: National Optical Astronomy Observatories)

Yong, D., Lambert, D. L., Allende Prieto, C., \& Paulson, D. B. 2004, ApJ, 603, 697

York, D. G., et al. 2000, AJ, 120, 1579 